\title{
Multiperiod Hierarchical Location Problem of Transit Hub in Urban Agglomeration Area
}

\author{
Ting-ting Li, Rui Song, Shi-wei He, Ming-kai Bi, Wei-chuan Yin, and Ying-qun Zhang
}

MOE Key Laboratory for Urban Transportation Complex Systems Theory and Technology,

Beijing Jiaotong University, Beijing 100044, China

Correspondence should be addressed to Ting-ting Li; rettyltt@foxmail.com and Rui Song; rsong@bjtu.edu.cn

Received 3 August 2016; Accepted 7 November 2016; Published 12 January 2017

Academic Editor: Aime’ Lay-Ekuakille

Copyright (C) 2017 Ting-ting Li et al. This is an open access article distributed under the Creative Commons Attribution License, which permits unrestricted use, distribution, and reproduction in any medium, provided the original work is properly cited.

With the rapid urbanization in developing countries, urban agglomeration area (UAA) forms. Also, transportation demand in UAA grows rapidly and presents hierarchical feature. Therefore, it is imperative to develop models for transit hubs to guide the development of UAA and better meet the time-varying and hierarchical transportation demand. In this paper, the multiperiod hierarchical location problem of transit hub in urban agglomeration area (THUAA) is studied. A hierarchical service network of THUAA with a multiflow, nested, and noncoherent structure is described. Then a multiperiod hierarchical mathematical programming model is proposed, aiming at minimizing the total demand weighted travel time. Moreover, an improved adaptive clonal selection algorithm is presented to solve the model. Both the model and algorithm are verified by the application to a reallife problem of Beijing-Tianjin-Hebei Region in China. The results of different scenarios in the case show that urban population migration has a great impact on the THUAA location scheme. Sustained and appropriate urban population migration helps to reduce travel time for urban residents.

\section{Introduction}

In the process of rapid urbanization in developing countries, regional spatial structure experiences tremendous changes, especially with the development of urban agglomeration area (UAA). On the one hand, because of the increasing close connection between cities in UAA, intercity passenger demand grows rapidly and presents hierarchical features from the aspects of travel distance and travel space. Also, more convenient transport service is required by passengers. On the other hand, the development of UAA is uncoordinated and meets challenges. More specifically, some megacities develop fast but face conflicts between their growing population and decreasing urban carrying capacity, while some small and medium-sized cities have low proportion of urban residents and their developments lag behind. Therefore, it is required to intensify the transportation network, promote the distribution of key industries and public resources, and shift away some of the megacities' functions and population, so as to help small and medium-sized cities to develop industries and attract residents to UAA.

In addition, it is demonstrated that transit hubs have the potential to drive the development of their surrounding areas. Thus, it is necessary to study the hierarchical location problem of transit hub in urban agglomeration area (THUAA), which contributes to accommodating the hierarchical feature of passenger demand in urban agglomeration area (PDUAA) and coordinating the development of UAA. Moreover, the location problem of THUAA is a long-term planning. Transportation network and passenger demand in the process of rapid urbanization are constantly changing. The hubs are hard to rebuild due to its huge resource consumption and political impacts. Considering all these, it is imperative to make overall plan for the multiperiod hierarchical location problem (MHLP) of THUAA.

MHLP of THUAA studied in this paper is a strategic planning. The planning horizon is divided into several time periods. It decides which hierarchy a city or an administrative 
district should be in each specific period. The goal is to maximize the accessibility of population to get transportation services in a UAA. Therefore, it does not involve the detailed location problem of stations.

The main contributions of this paper are (i) a hierarchical service network of THUAA with a multiflow, nested and noncoherent structure; (ii) a mathematical programming model and a solution algorithm for MHLP of THUAA; and (iii) application to a real-life problem in China to meet changes in the process of rapid urbanization.

The remainder of this paper is organized as follows. Section 2 reviews the related hierarchical and multiperiod facility location problem literatures. Section 3 defines the hierarchical service network of THUAA. Section 4 presents the optimization model. Section 5 proposes a solution method to solve the model. In Section 6 the model is applied to a reallife case of Beijing-Tianjin-Hebei Region in China. Finally, main findings and future work directions are summarized in Section 7.

\section{Literature Review}

In the context of rapid urbanization, the system of THUAA is multilevel so as to provide hierarchical service for various passengers. However, the hierarchical feature of THUAA system has been neglected in most researches. Furthermore, the multiperiod nature of the hierarchical location problem of THUAA attracted few attention. According to the previous definitions, the location problem of THUAA can be viewed as a facility location problem. Therefore, the hierarchical and multiperiod facility location models can be applied to MHLP of THUAA, which are reviewed as follows.

There has been a rich body of literatures about hierarchical facility location problem (HFLP), as seen in the recent reviews $[1,2]$. The HFLP models are now mainly employed in the following areas: health care system [3-5], education system [6], disaster management $[7,8]$, solid waste management [9], and telecommunications network [10, 11], and so forth. However, the HFLP models have not been applied in the location problem of THUAA. Exact solution methods, such as branch-and-bound [12], are utilized to solve HFLPs. With the increasing size and complexity of real-life HFLPs, nonexact solution methods have also been proposed (e.g., approximation algorithm [13, 14], tabu search [15]).

As a kind of dynamic facility location models (see the review [16]), multiperiod facility location model divides the planning horizon into several time periods, which helps to better deal with the time-varying parameters [17]. Till now, a large number of researches have been done on multiperiod facility location models [18-21]. However, there are few literatures involving multiperiod HFLP models. Hinojosa et al. [22] presented a mix integer programming model to address the location-allocation problem of the production plants and intermediate warehouses in different time periods. The model was solved by Lagrangian relaxation combined with a heuristic procedure. Wang et al. [23] addressed the location problem of regional distribution centers and stores in a distribution system across several time periods. A genetic

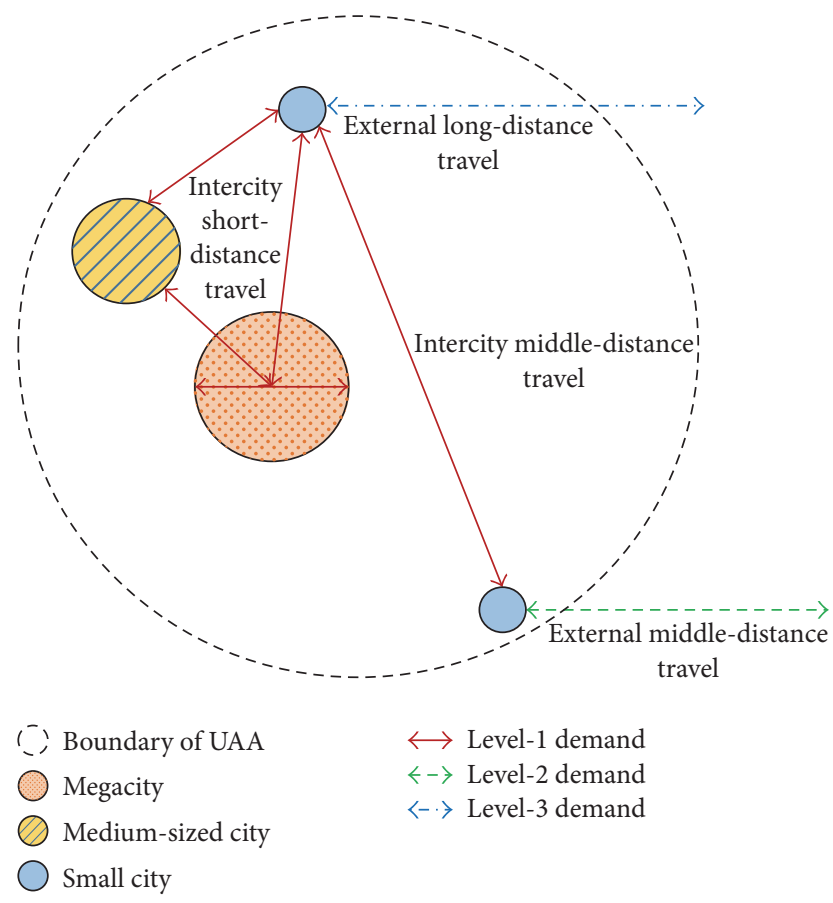

FIGURE 1: The hierarchical structure of PDUAA.

algorithm based heuristic was utilized to solve the problem. Antunes et al. [24] proposed a multiperiod hierarchical location model for urban hierarchy planning, aiming at minimizing the total demand weighted travel time for the residents to get service from different-level facilities during several time periods. Pehlivan et al. [3] studied the adjustment of hierarchical perinatal network, that is, when and where to locate different-level maternity facilities. The objective of the model is to minimize the total cost. Also, the service quality of each facility is ensured above a given level.

The preceding analyses show that multiperiod HFLP modeling needs more researches and the solution methods for solving large-size problems in realistic situations should be paid more attention. Therefore, the application of multiperiod HFLP models to MHLP of THUAA is innovative and has practical significance.

\section{Problem Description}

In the process of rapid urbanization, PDUAAs form a hierarchical system (see Figure 1), and so are the THUAAs (see Figure 2). In addition, different-level THUAAs serve different-level PDUAAs (see Figure 2).

The hierarchical structure of PDUAA is presented in Figure 1. According to the travel distance and the travel space, PDUAAs are divided into 3 levels of demands, in which the intercity short-distance and middle-distance travel are called level-1 demand, the external middle-distance travel is called level-2 demand, and the external long-distance travel is called level-3 demand.

Figure 2 presents the hierarchical structure of THUAA and the demands served by different-level hubs. THUAA 


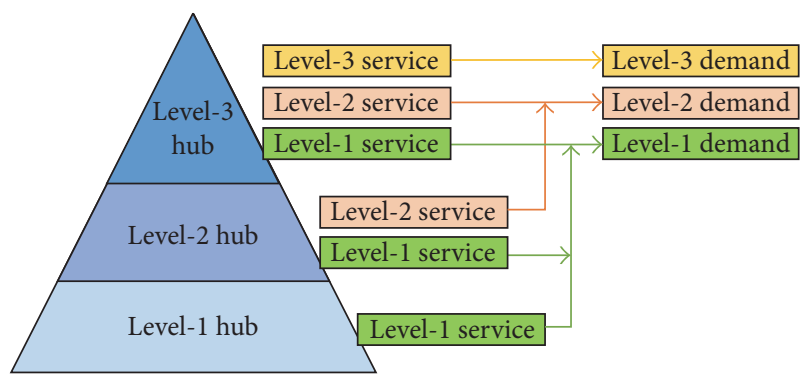

FIGURE 2: The hierarchical structure of THUAA and the demands served by different-level hubs.

system consists of 3 levels of hubs. Different-level hubs provide different-level services. More specifically, level-3 hub provides all services (i.e., it can serve all demands). Level2 hub provides level-2 service and level-1 service (i.e., it can serve level-2 demand and level-1 demand). Level-1 hub provides level-1 service (i.e., it can only serve level-1 demand).

The essence of MHLP of THUAA can be explained as follows: (i) in a given UAA, the transportation network and passenger demand are known, and the planning horizon is divided into several time periods; (ii) a hub can only serve the demands in its coverage area; and (iii) the target is to choose appropriate administrative districts or cities for different-level hubs in each period, thus satisfying differentlevel demands in UAA and minimizing the total demand weighted travel time. As shown in Figure 3, the yellow point, the red point, the blue point, and the gray point represent level-3 hub, level-2 hub, level-1 hub, and demand point, respectively. The coverage area of a hub is marked with a dashed line circle.

According to the previous descriptions, the hierarchical location problem of THUAA is a HFLP with a multiflow, nested, and noncoherent structure (see Figure 4). It is a multiflow system because any lower-level demand can be assigned to any higher-level hub. For instance, level-1 demand of $\mathrm{C} 1$ is assigned to a level-2 hub B2. Because higher-level hubs provide more services than lower-level hubs, it is nested. Since the structure is noncoherent, the demand points served by the same hub for their lower-level demands may be served by different hubs for their higher-level demands. For instance, level-1 demands of $\mathrm{B} 0$ and $\mathrm{C} 0$ are both assigned to $\mathrm{B} 1$, while level-3 demands of $\mathrm{B} 0$ and $\mathrm{C} 0$ are assigned to $\mathrm{A} 3$ and $\mathrm{B} 3$, respectively.

\section{Modeling}

4.1. Notation. In order to formulate the model, the following notations are defined.

Sets

$H$ : the set of demand levels (or set of service levels or set of hub levels), $H=\{1,2,3\}$

$I$ : the set of demand points

$J$ : the set of candidate hubs

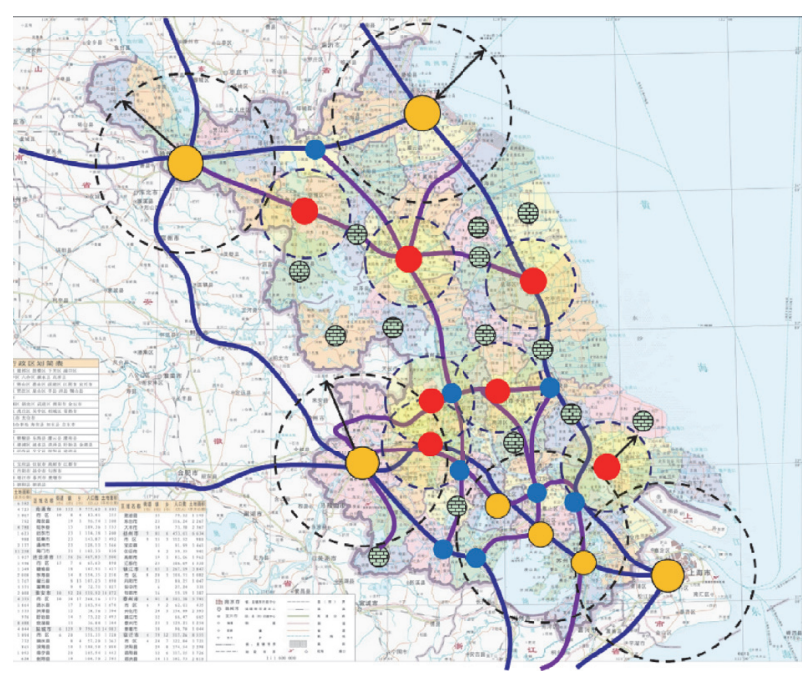

FIgURE 3: The concept about hierarchical location problem of THUAA.

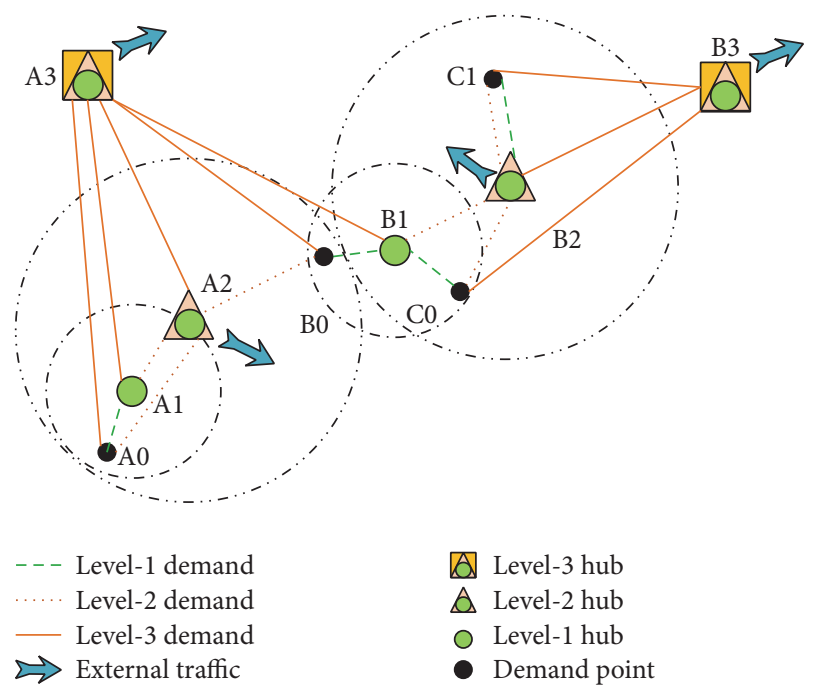

FIGURE 4: The hierarchical service network of THUAA with a multiflow, nested and noncoherent structure.

$T$ : the set of periods, $T=\{1,2,3\}$. More specifically, periods 1,2 , and 3 represent the short-term period, the medium-term period, and the long-term period, respectively

\section{Parameters}

$L_{i j}$ : the line distance between demand point $i$ and candidate hub $j$

$d_{i j t}$ : the shortest travel time between demand point $i$ and candidate hub $j$ in period $t$

$q_{\text {ist }}$ : level-s demand of demand point $i$ in period $t$

$c_{h}$ : the minimum quantity of level- $h$ demand served by a level- $h$ hub 
$C_{j s t}$ : the maximum capacity of level-s service provided by candidate hub $j$ in period $t$

$R_{h}$ : the coverage of level- $h$ hub

\section{Decision Variables}

$x_{i j s t}$ : let $x_{i j s t}$ be one if level-s demand of demand point $i$ is served by hub $j$ in period $t$, zero otherwise.

$y_{j h t}$ : let $y_{j h t}$ be one if candidate hub $j$ is opened as a level- $h$ hub in period $t$, zero otherwise.

\subsection{Assumptions}

(1) For a demand point, demands at each level are served by only one hub.

(2) The coverage of hubs at each level and the planning periods are specific.

(3) The transportation network and different-level demands of each demand point in each period are known.

(4) The minimum quantity of the highest-level demand served by different-level hubs is a known constant.

(5) The maximum capacities of different-level services provided by each candidate hub in each period are limited and known.

4.3. Mathematical Formulation. The multiperiod hierarchical location model of THUAA is formulated as follows:

$$
\begin{aligned}
& \text { Minimize } \sum_{i \in I} \sum_{j \in J} \sum_{s \in H} \sum_{t \in T} d_{i j t} q_{i s t} x_{i j s t} \\
& \text { Subject to: } \sum_{j \in J} x_{i j s t}=1 \quad \forall i \in I, s \in H, t \in T \\
& \sum_{h \in H} y_{j h t} \leq 1 \quad \forall j \in J, t \in T \\
& x_{i j s t} \leq \sum_{h \in H \mid h \geq s} y_{j h t} \\
& \forall i \in I, j \in J, s \in H, t \in T \\
& \sum_{i \in I} q_{i h t} x_{i j h t} \geq c_{h} y_{j h t} \\
& \forall j \in J, h \in H, t \in T \\
& \sum_{i \in I} q_{i s t} x_{i j s t} \leq \sum_{h \in H \mid h \geq s} C_{j s t} y_{j h t} \\
& \forall j \in J, s \in H, t \in T \\
& \sum_{h \in H \mid h \geq s} y_{j h t} R_{h} \geq x_{i j s t} L_{i j} \\
& \forall i \in I, j \in J, s \in H, t \in T \\
& y_{j h(t-1)} \leq \sum_{k \in H \mid k \geq h} y_{j k t} \\
& \forall j \in J, h \in H, t \in\{2,3\}
\end{aligned}
$$

$$
\begin{aligned}
& x_{i j s t} \in\{0,1\}, \\
& y_{j h t} \in\{0,1\} \\
& \quad \forall i \in I, \quad j \in J, h \in H, s \in H, \quad t \in T .
\end{aligned}
$$

The objective function (1) minimizes the total demand weighted travel time in the planning horizon. Constraints (2) ensure that all demands in each period are satisfied. Constraints (3) represent that different-level hubs cannot be located at the same candidate hub in each period. Constraints (4) impose that demands at each level can only be served by a hub of equal or higher level in each period. Constraints (5) enforce that the highest-level demand served by a hub in each period exceeds the minimum quantity. Constraints (6) state that different-level demands served by a hub in each period do not exceed the maximum capacity. Constraints (7) limit that a hub can only serve the demand points in its coverage area. Constraints (8) guarantee that a hub cannot be downgraded once it is opened. Constraints (9) are the binary constraints for the variables.

\section{Solution Method: Improved Adaptive Clonal Selection Algorithm (IACSA)}

Adaptive clonal selection algorithm (ACSA) $[25,26]$ is widely used because of its fast convergence. Simulated annealing (SA) [27] is a parallel and global optimization algorithm, not depending on the initial population. Considering the feature of the encoding and decoding for the MHLP model, improved adaptive clonal selection algorithm (IACSA) is put forward, in which the idea of SA is introduced into ACSA, thus ensuring global optimization and fast convergence.

5.1. The Procedure of IACSA. In immunology, clone means asexual propagation. A clone refers to one or more offspring derived from a single ancestor, whose genetic composition is identical to that of the ancestor. In IACSA, (i) an antibody represents a solution; (ii) affinity measures the fitness of an antibody; and (iii) antibody population is a group of solutions. As is shown in Figure 5, the steps of IACSA are as follows.

Step 1 (initialization). $M$ feasible antibodies are generated randomly, composing the initial antibody population $P$.

Step 2 (evaluation). The affinities of the antibodies in $P$ are calculated according to the affinity function and then are sorted in descending order.

Step 3 (clonal proliferation). Each antibody in $P$ is cloned and then an antibody population $C$ is formed.

Step 3.1. The antibody in $P$ is cloned by neighborhood search. The clonal factor of an antibody (i.e., the number of clones created for an antibody) is proportional to its affinity. Namely, an antibody with a higher affinity will have a higher clonal factor. 


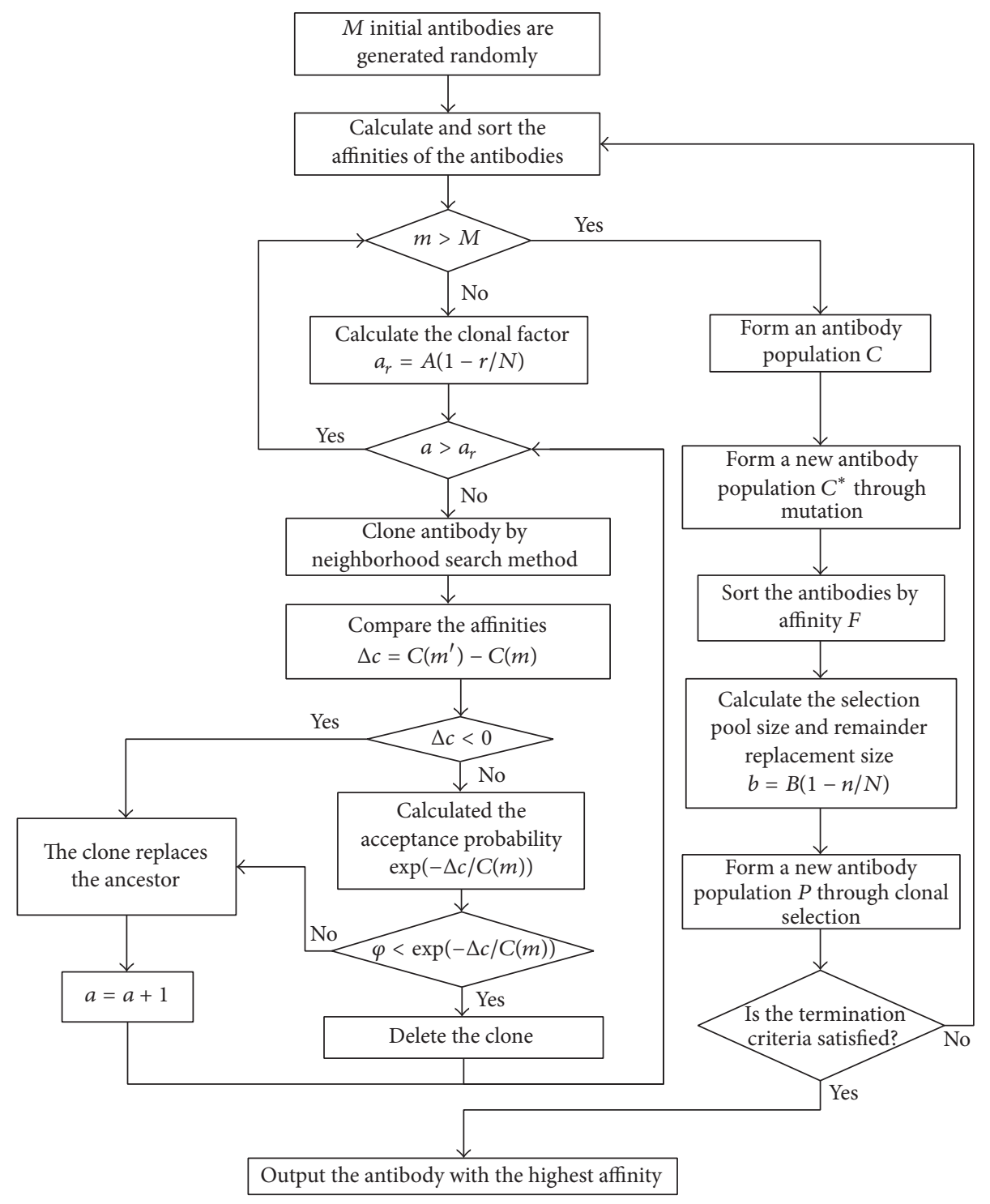

FIgURE 5: The procedure of IACSA.

Step 3.2. $\Delta c=C\left(m^{\prime}\right)-C(m)$ is defined to compare the affinity of a clone $C\left(m^{\prime}\right)$ with that of its ancestor $C(m)$. Specifically, the idea of SA is introduced to use probability proportion to accept the worse clones, thus expanding the search space and preventing premature. According to the Metropolis acceptance criteria (i.e., an acceptance theory based on probability): if $\Delta c \leq 0$, the ancestor is replaced by the clone; otherwise, the clone is accepted with probability $\exp (-\Delta c / C(m))$.

Step 3.3. Steps 3.1 and 3.2 are repeated $M$ times to form an antibody population $C$.

Step 4 (hypermutation). A new antibody population $C^{*}$ is generated by the hypermutation operation.

Step 5 (clonal selection). Select the antibodies whose affinities rank in the top $b$ from $C^{*}$ to replace the antibodies whose affinities rank in the bottom $b$ in $P$.
Step 6 (termination test). If termination criterion is met, stop and output the antibody with the highest affinity in $P$; otherwise, go to Step 3 .

5.2. The Detail of IACSA. The detailed design of IACSA is as follows.

(1) Encoding and Decoding. Integer encoding is used. Each antibody is composed of 9 fragments. Each fragment of an antibody represents the serial numbers of hubs which serve different-level demands of each demand point in each period. For example, in Figure 6, level-1 and level-2 demands of demand point 1 are both served by hub 1 in period 1 , while level- 3 demand of demand point 1 is served by hub 2 in period 1.

Moreover, each antibody is divided into 3 parts by period. The hub level in each period can be judged by each part of the antibody. Firstly, a hub which serves level-3 demand is judged as a level-3 hub. Secondly, a hub serves level-2 demand but no 


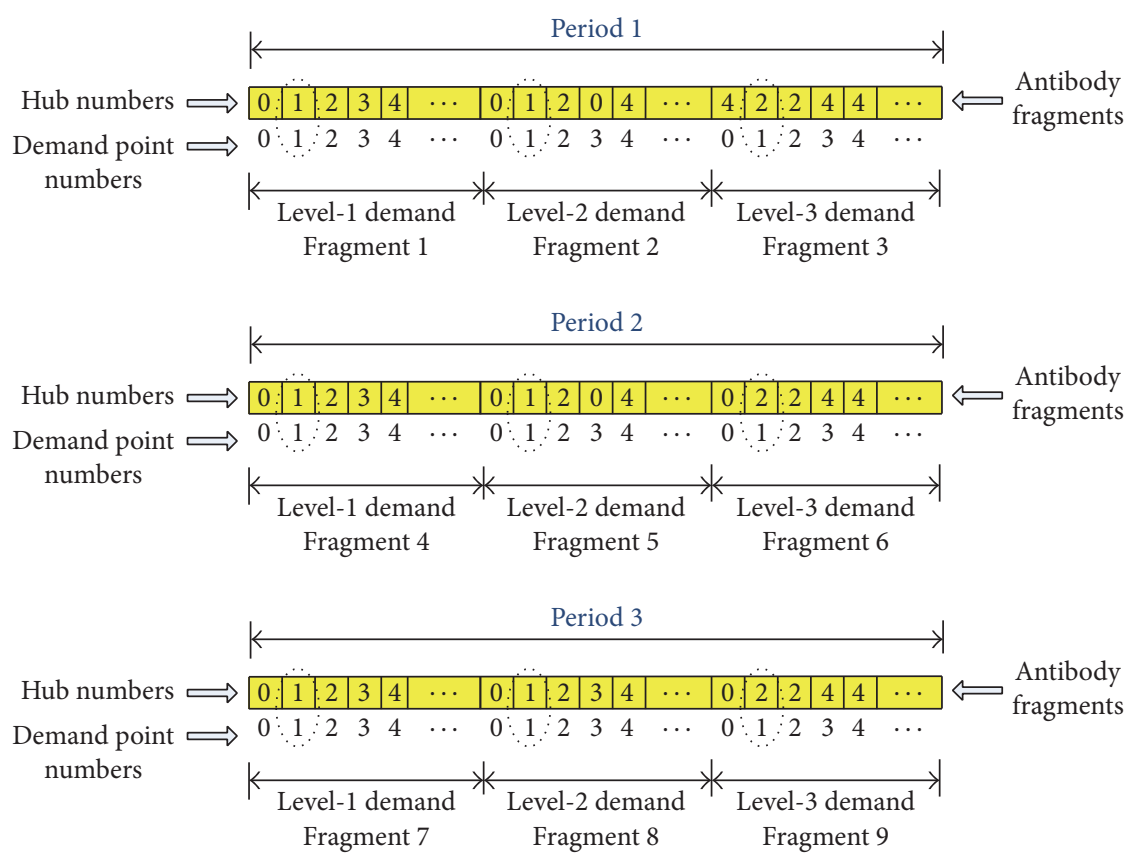

FIGURE 6: Encoding of solution.
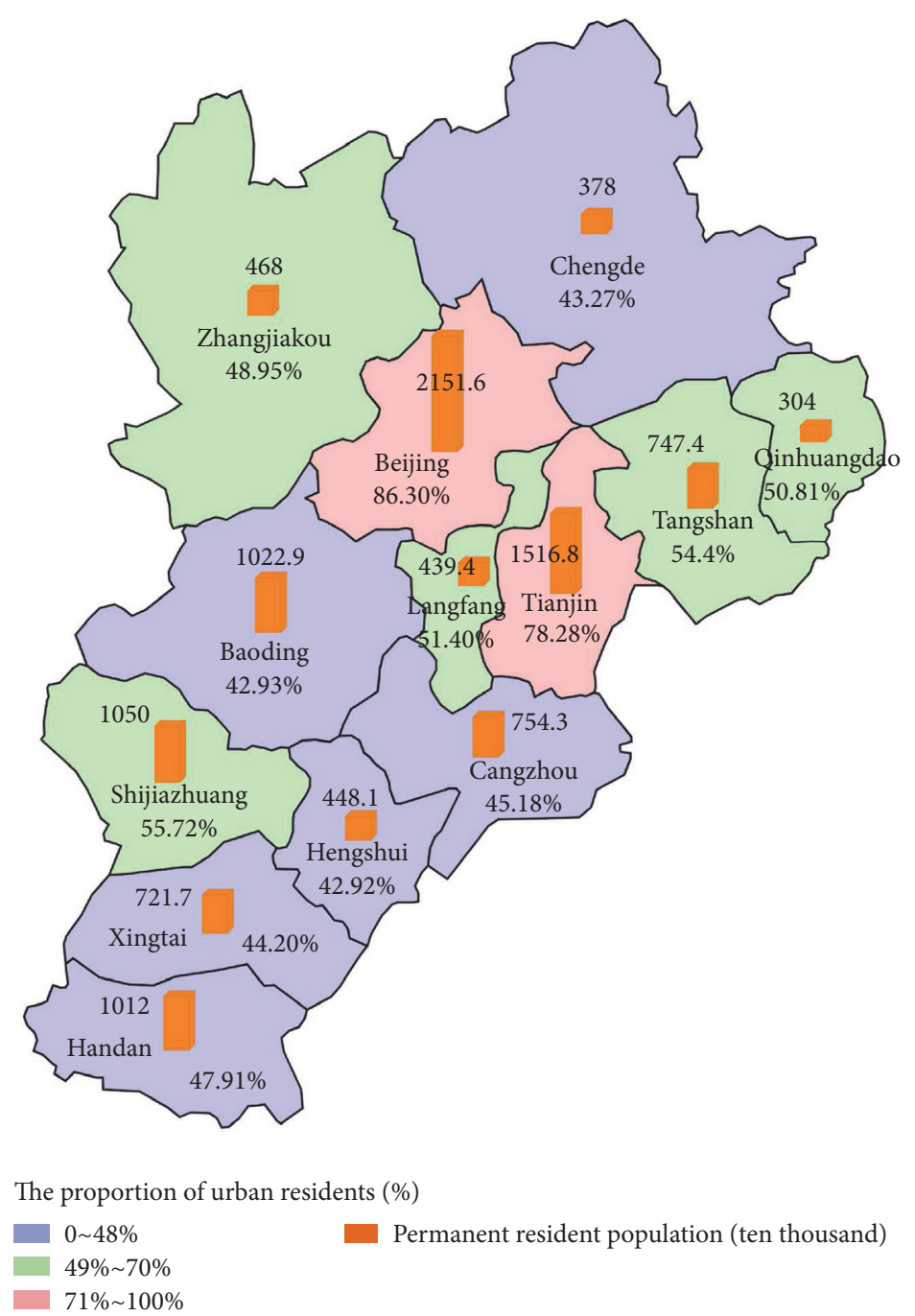

Figure 7: The population and urban residents proportion of cities in Beijing-Tianjin-Hebei Region in 2014. 


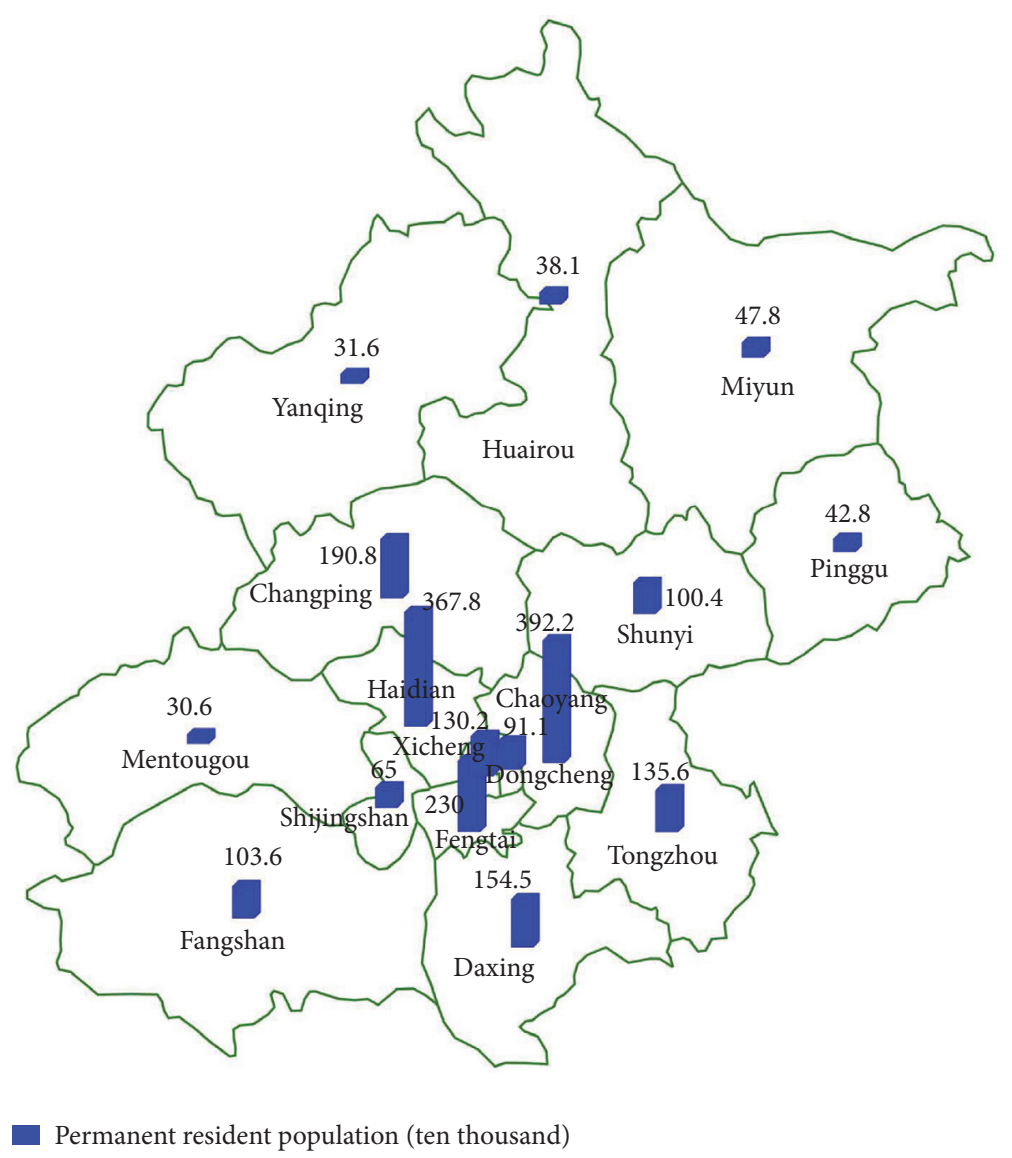

FIGURE 8: The population of each administrative district in Beijing in 2014.

level-3 demand is judged as a level-2 hub. Finally, a hub serves only level-1 demand is judged as a level-1 hub. For instance, Figure 6 shows that (i) in period 1, hubs 2 and 4 are both level3 hubs, while hubs 0 and 1 are both level- 2 hubs and hub 3 is a level-1 hub; (ii) in period 2, hubs 0,2 , and 4 are all level-3 hubs, while hub 1 and hub 3 are level- 2 hub and level-1 hub, respectively; and (iii) in period 3, hubs 0,2 , and 4 are all level3 hubs, while hubs 1 and 3 are both level-2 hubs.

(2) Initialization. Because different-level demands are served by different-level hubs, each gene of the antibody is generated according to the corresponding candidate hub set. More specifically, level-3 demand can be served by level- 3 candidate hubs. Level-2 demand can be served by level-2 and level3 candidate hubs. Level-1 demand can be served by all candidate hubs. Firstly, a candidate hub is selected randomly from the corresponding candidate hub set for each gene in period 1 . Then the antibody fragments of period 1 are copied to periods 2 and 3, respectively. Thus, an initial antibody is formed.

(3) Affinity Function. According to the above encoding and initialization method, constraints (2), (3), (4), and (9) are all satisfied. Constraints (8) are judged whether it is satisfied when a new antibody is generated. Therefore, constraints
(5), (6), and (7) are dealt with the penalty function method, adding to the objective function. $Q$ is a large constant. $\delta_{1}, \delta_{2}$, and $\delta_{3}$ are penalty factors. The affinity function $F$ is shown in

$$
\begin{aligned}
F & =Q\left\{\sum_{i \in I} \sum_{j \in J} \sum_{s \in H} \sum_{t \in T} d_{i j t} q_{i s t} x_{i j s t}\right. \\
& +\delta_{1} \sum_{j \in J} \sum_{h \in H} \sum_{t \in T} \max \left\{0, c_{h} y_{j h t}-\sum_{i \in I} q_{i h t} x_{i j h t}\right\} \\
& +\delta_{2} \sum_{j \in J} \sum_{s \in H} \sum_{t \in T} \max \left\{0, \sum_{i \in I} q_{i s t} x_{i j s t}-\sum_{h \in H \mid h \geq s} C_{j s t} y_{j h t}\right\} \\
& \left.+\delta_{3} \sum_{i \in I} \sum_{j \in J} \sum_{s \in H} \sum_{t \in T} \max \left\{0, x_{i j s t} L_{i j}-\sum_{h \in H \mid h \geq s} y_{j h t} R_{h}\right\}\right\}^{-1} .
\end{aligned}
$$

(4) Clonal Factor. The clonal factor of the antibody whose affinity ranks number $r$ is defined as $a_{r}=A(1-r / N)$. Moreover, it is limited that $a_{r} \geq a_{0}$. A and $a_{0}$ are the maximum and minimum clonal factors, respectively. $N$ is the maximum number of iterations. 


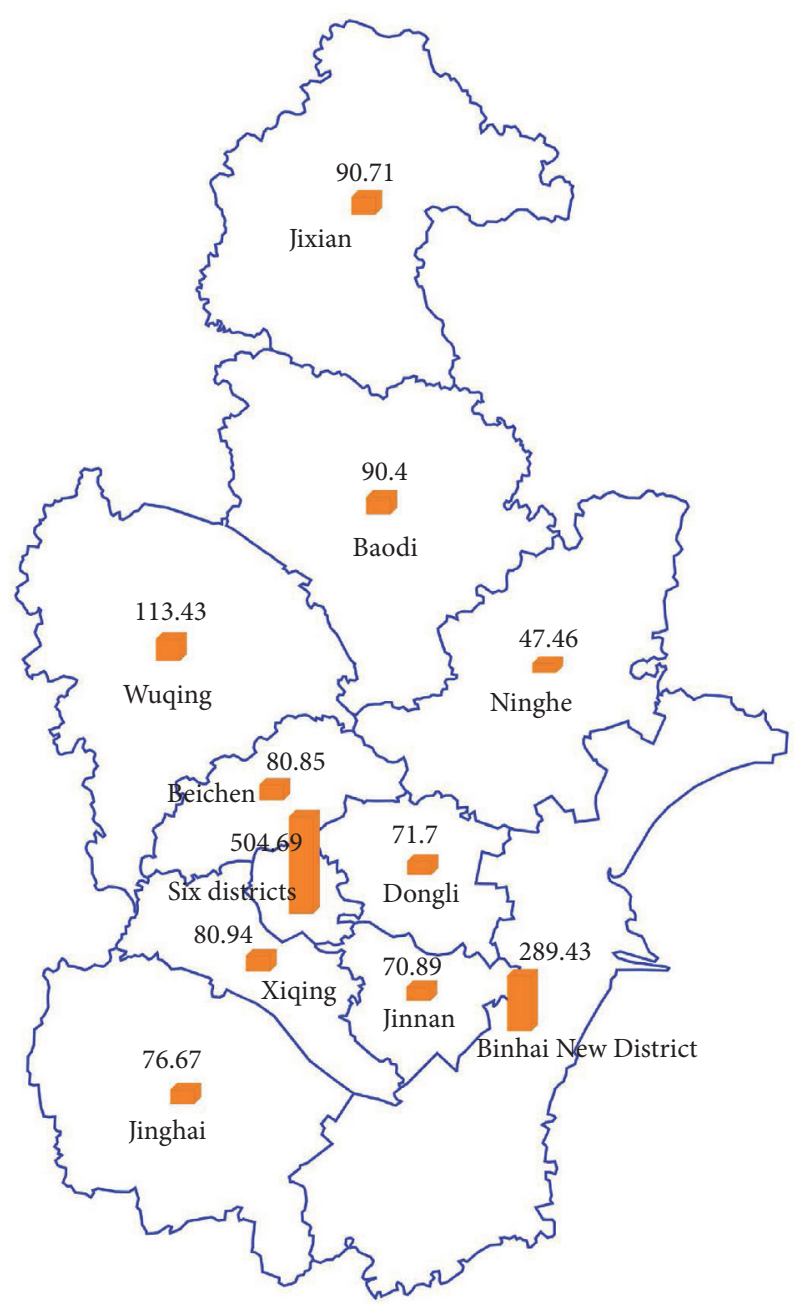

Permanent resident population (ten thousand)

FIGURE 9: The population of each administrative district in Tianjin in 2014.

(5) Mutation Rate. The mutation rate is defined as $\varepsilon=\varepsilon_{\max }(1-$ $n / N)$. Moreover, it is limited that $\varepsilon>\varepsilon_{\min } \cdot \varepsilon_{\max }$ and $\varepsilon_{\min }$ are the maximum and minimum mutation rates, respectively. $n$ is the number of current iterations. $N$ is the maximum number of iterations.

(6) Mutation Operation. A gene in each fragment of an antibody is selected randomly. A random number $\lambda$ between 0 and 1 is generated. The current gene is replaced if $\lambda<\varepsilon$; otherwise it remains itself.

(7) Selection Pool Size and Remainder Replacement Size. Selection pool size and remainder replacement size are both defined as $b$. More specifically, $b=B(1-n / N)$. Moreover, it is limited that $b>b_{0} . B$ and $b_{0}$ are the maximum and minimum sizes, respectively. $n$ is the current iteration. $N$ is the maximum number of iterations.

(8) Termination Criteria. The algorithm terminates when the number of iterations reaches the predefined maximum number.

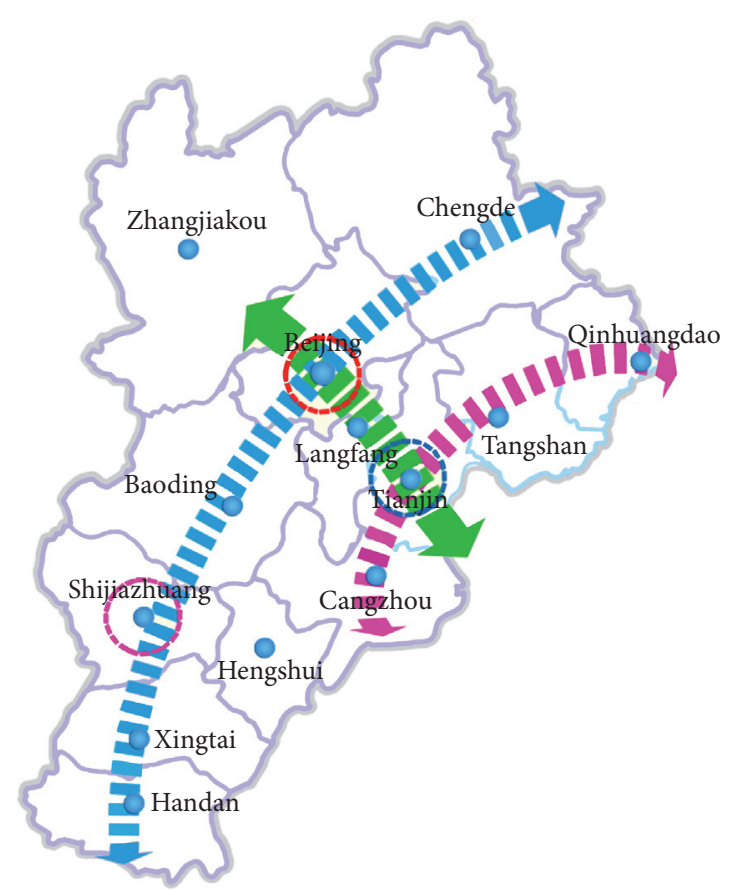

FIGURE 10: The spatial development pattern of Beijing-Tianjin-Hebei Region.

\section{Case Study}

This section applies the model to a real-life problem of Beijing-Tianjin-Hebei Region in China. Beijing-TianjinHebei Region is an important UAA in China. However, the development of Beijing-Tianjin-Hebei Region is not coordinated enough. In Figure 7, the population and urban residents proportion of cities in Beijing-Tianjin-Hebei Region in 2014 are presented, which show that the population and urban residents proportion of Beijing and Tianjin are much higher than those of other cities.

Figure 8 presents the population of each administrative district in Beijing in 2014. It can be seen that the population in the central area is much higher than that of suburban districts. More specifically, Beijing is facing great pressure of population, which needs functional dispersal and decentralization population in the future. Figure 9 presents the population of each administrative district in Tianjin in 2014. The center of Tianjin has high population density, while the population of Binhai and other suburban areas in Tianjin are small.

In the future, Beijing-Tianjin-Hebei Region will be developed in the light of the spatial pattern presented in Figure 10. Moreover, intercity rail will be constructed according to the planning described in Figure 11.

6.1. Data. The administrative districts in Beijing (see Figure 8), the administrative districts in Tianjin (see Figure 9), and the other 11 cities in Beijing-Tianjin-Hebei Region (see Figure 7) are considered as both demand points and candidate hubs. Periods 1 3 represent the short-term period (year 


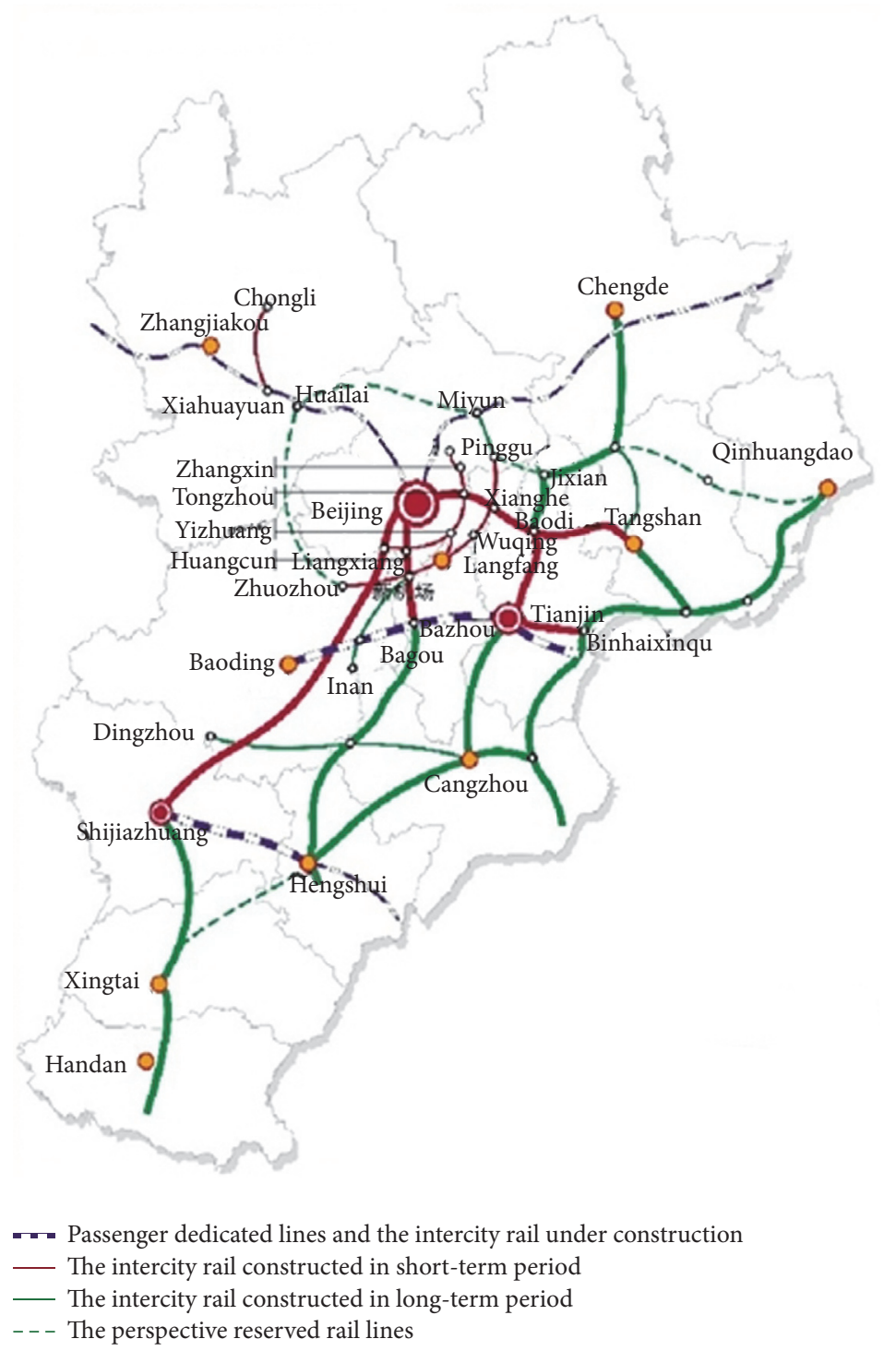

FIGURE 11: Intercity rail planning of Beijing-Tianjin-Hebei Region (years 2014 2030).

TABLE 1: The results comparison between IACSA and CPLEX.

\begin{tabular}{|c|c|c|c|c|c|}
\hline \multirow{2}{*}{ The number of areas in UAA } & \multicolumn{3}{|c|}{ Objective function value } & \multicolumn{2}{|c|}{ Computational time (s) } \\
\hline & $Z_{\text {CPLEX }}$ & $Z_{\text {IACSA }}$ & Gap (\%) & CPLEX & IACSA \\
\hline 38 & 83523 & 83635 & 0.1 & 952.6 & 12.3 \\
\hline 300 & 3652384 & 3681603 & 0.6 & 42485.7 & 1845.1 \\
\hline
\end{tabular}

2015 2020), the medium-term period (year 2020 2025), and the long-term period (year 2025 2030), respectively. The data source and the value of the parameters are shown as follows:

$L_{i j}$ : it is measured in arcGIS; see Table 2 in Appendix. $R_{h}: R_{1}$ is defined as $70 \mathrm{~km} . R_{2}$ is defined as $150 \mathrm{~km} . R_{3}$ is defined as $300 \mathrm{~km}$.

$c_{h}$ : it is estimated according to the passenger transport volume of Beijing-Tianjin-Hebei Region from China's Urban Statistical Yearbooks in the recent years. $c_{1}$ is defined as 7000 thousand persons annually. $c_{2}$ is defined as 3000 thousand persons annually. $c_{3}$ is defined as 2500 thousand persons annually.

$d_{i j t}$ : considering the intercity rail planning presented in Figure 11, it is calculated by arcGIS. For example, the travel time between areas in period 1 is presented in Table 3 in Appendix.

$q_{i s t}$ : considering the population migration in the future and the increase of urban residents, it is reckoned by the population and trip frequency. For 


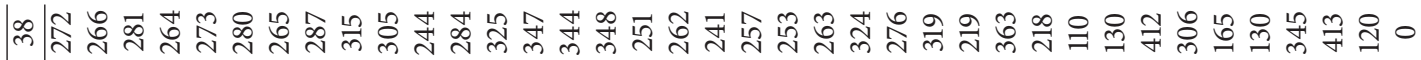

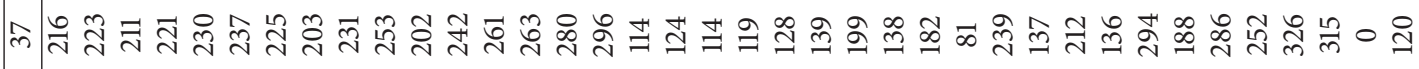

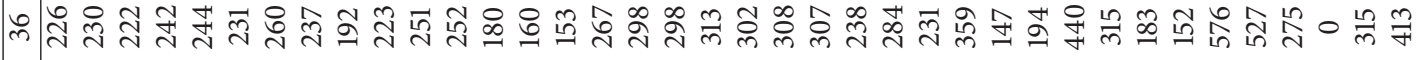

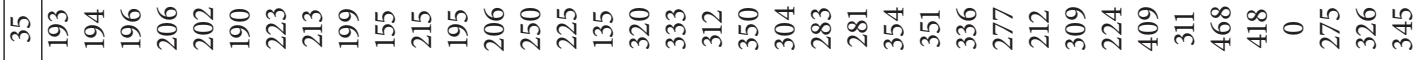

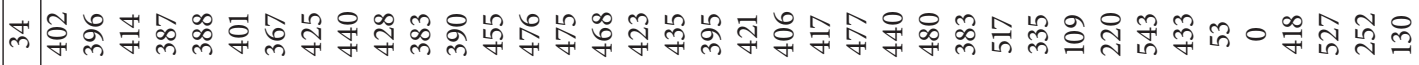

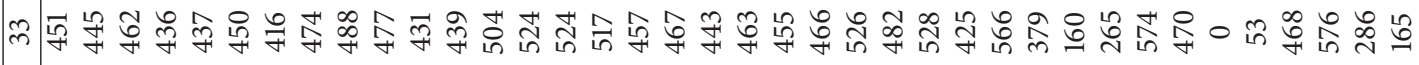

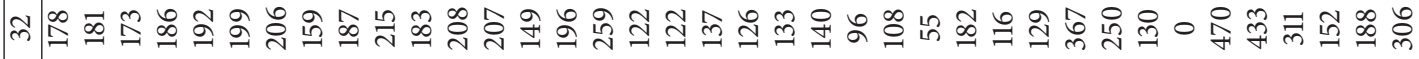

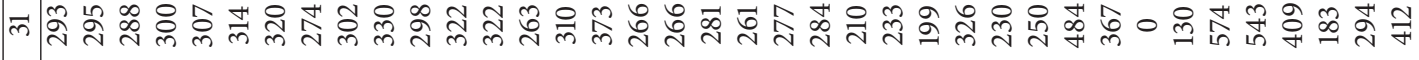

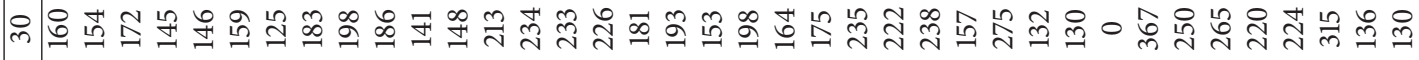

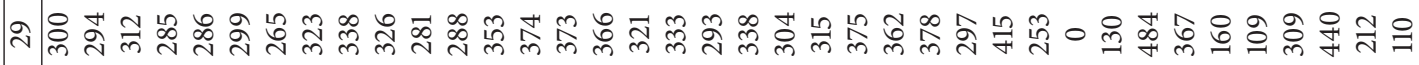

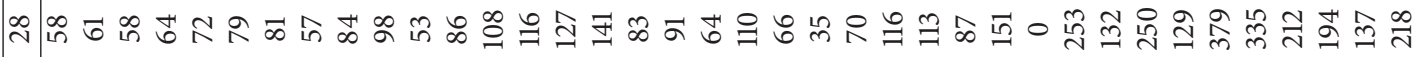

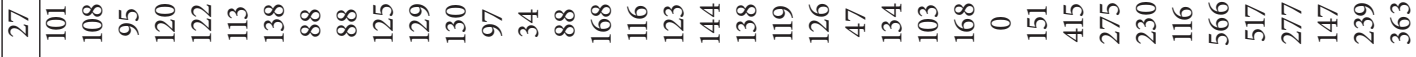

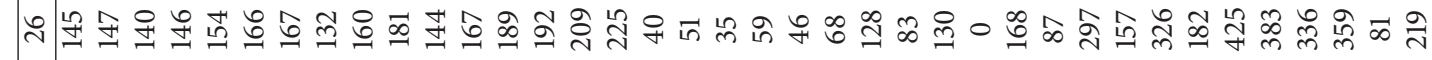

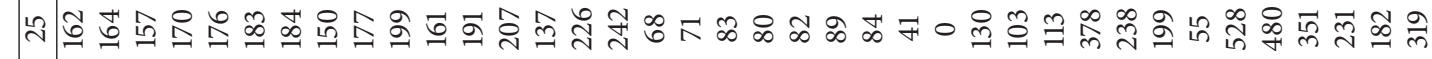

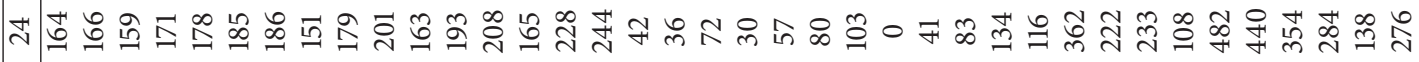

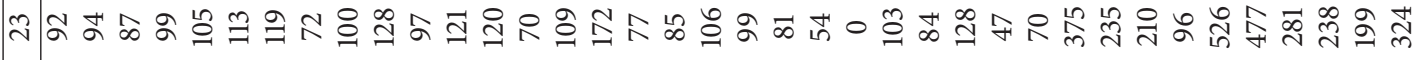

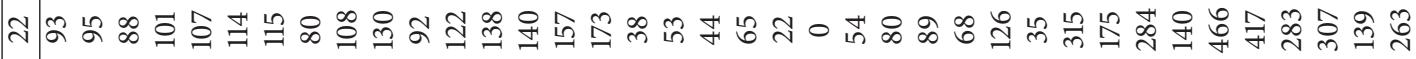

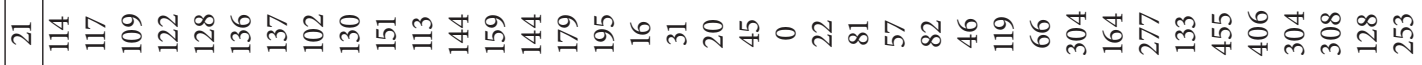

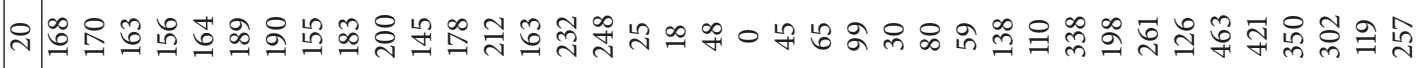

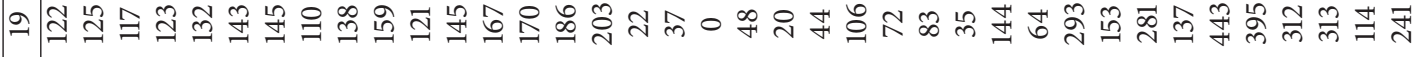

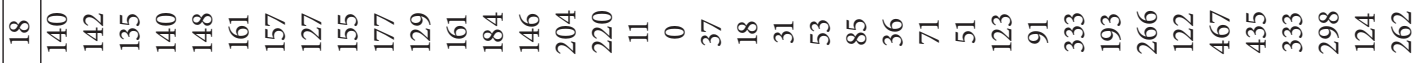

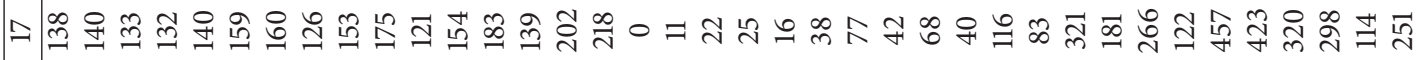

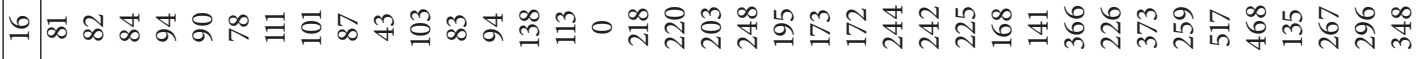

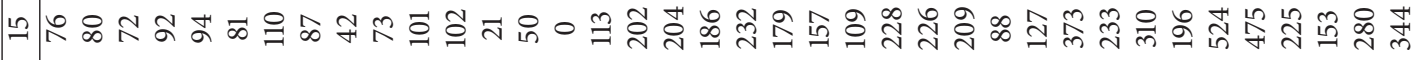

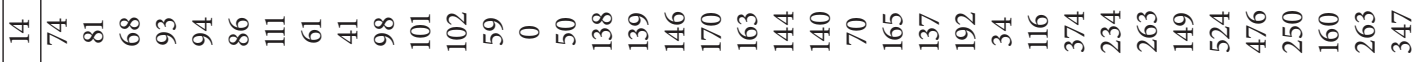

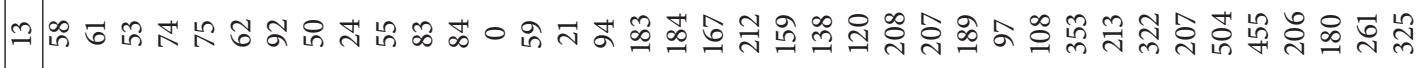

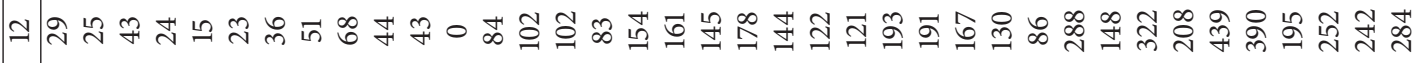

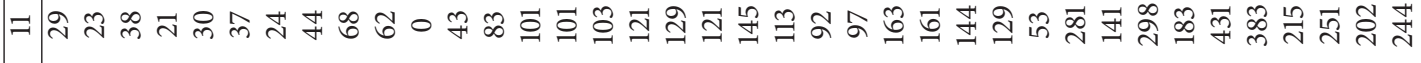

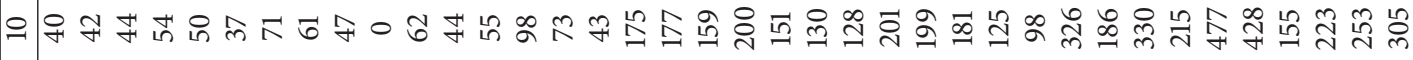

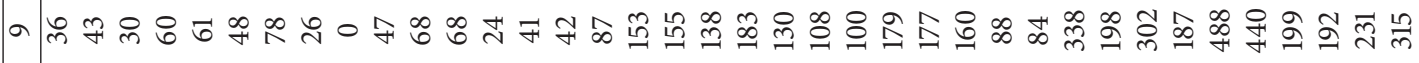

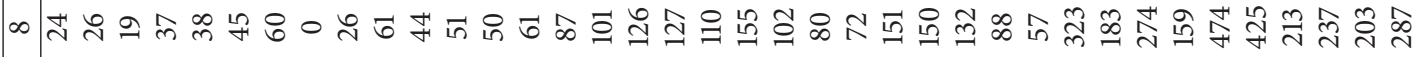

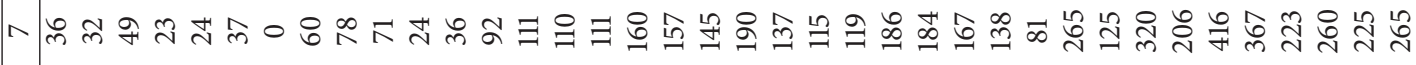

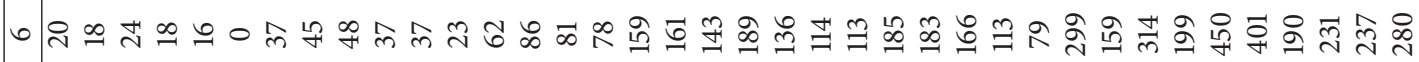

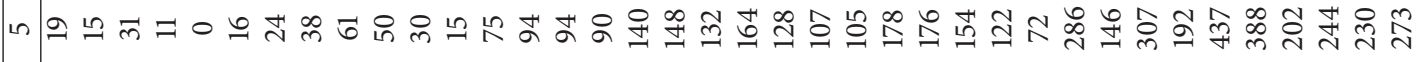

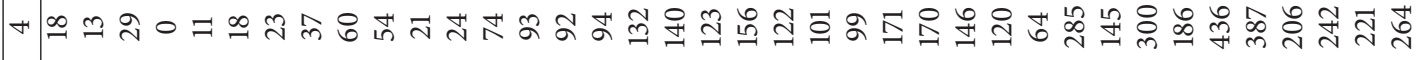

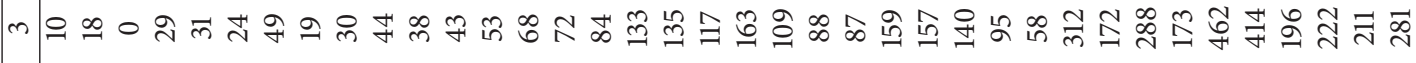
小

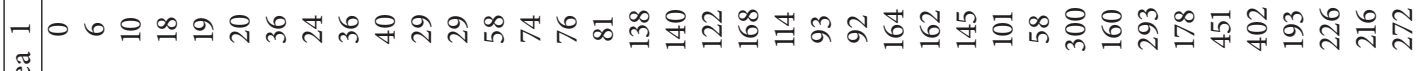

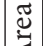

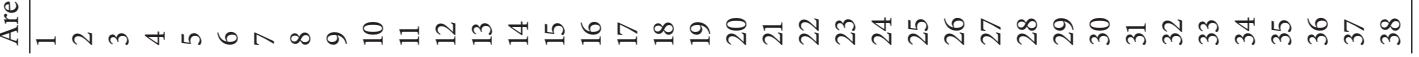




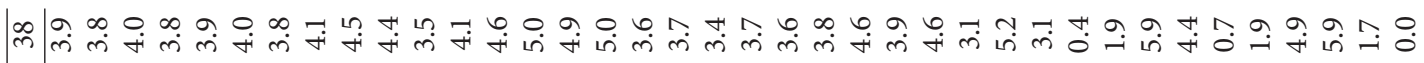

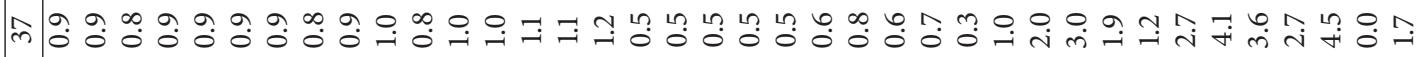

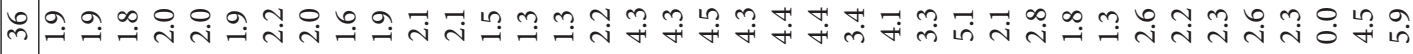
m m車

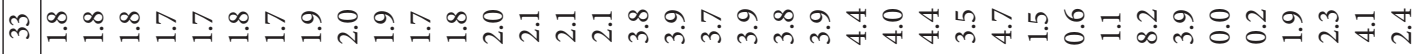

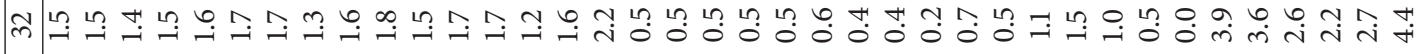

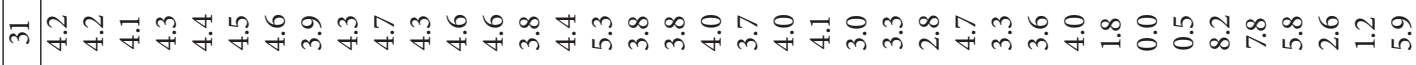

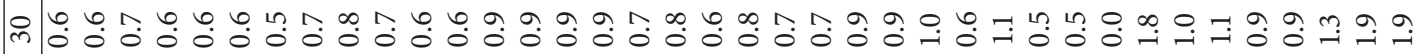

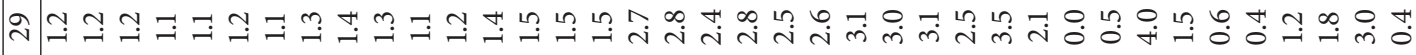

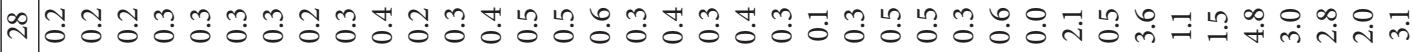

৯

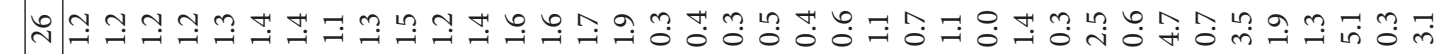

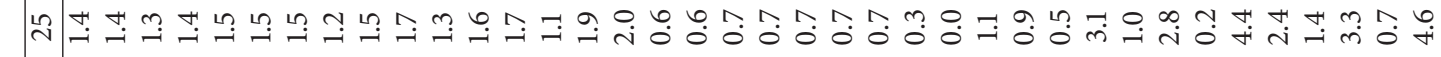

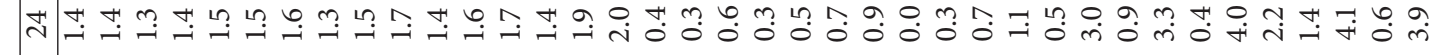

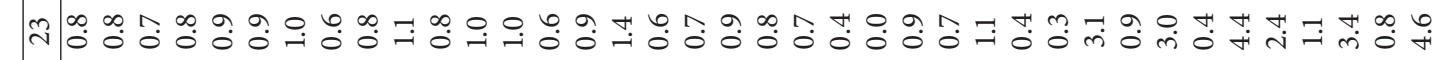

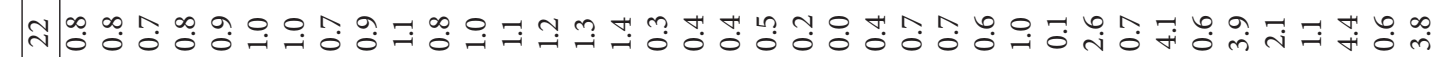
ה우엉

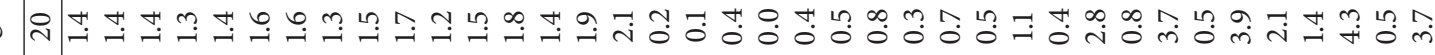

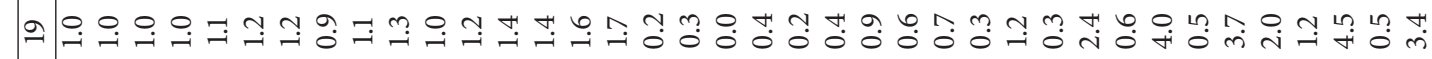

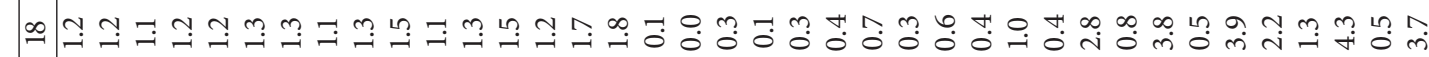

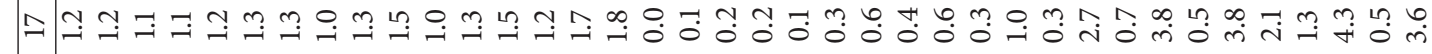

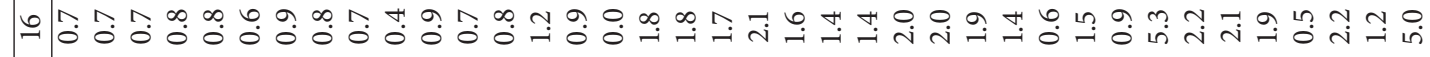

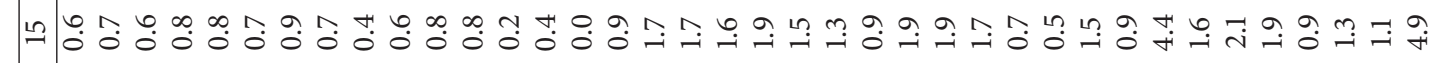

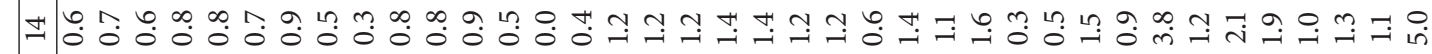
$\leadsto$ 눙

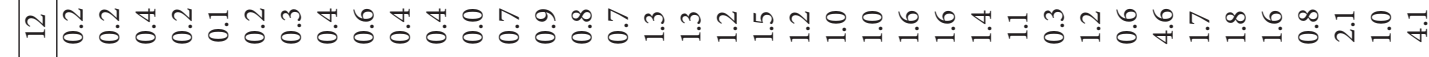

$=$ = స

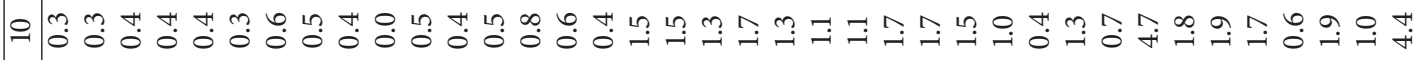

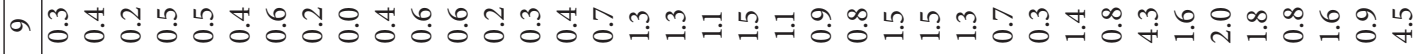

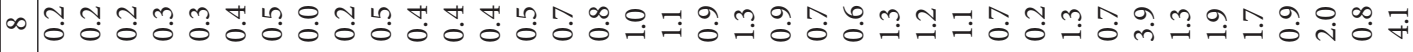

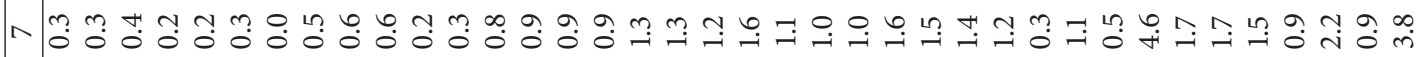

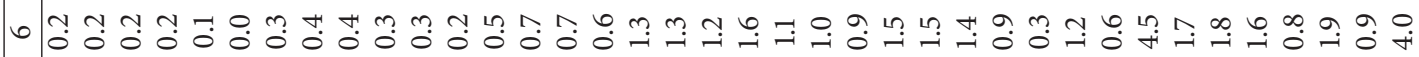

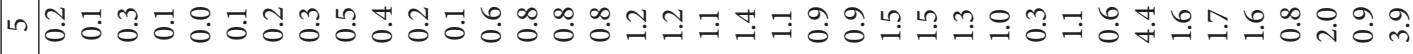

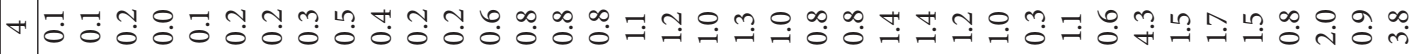

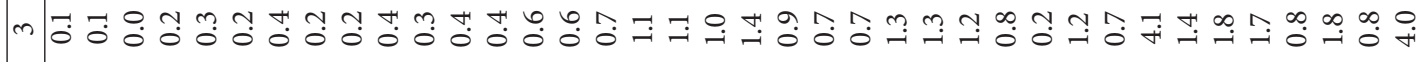
N

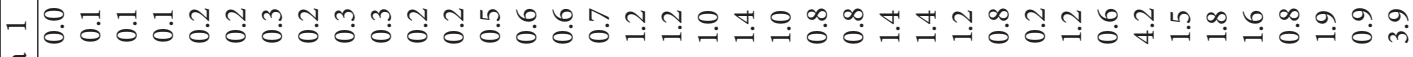
Ð્ป 


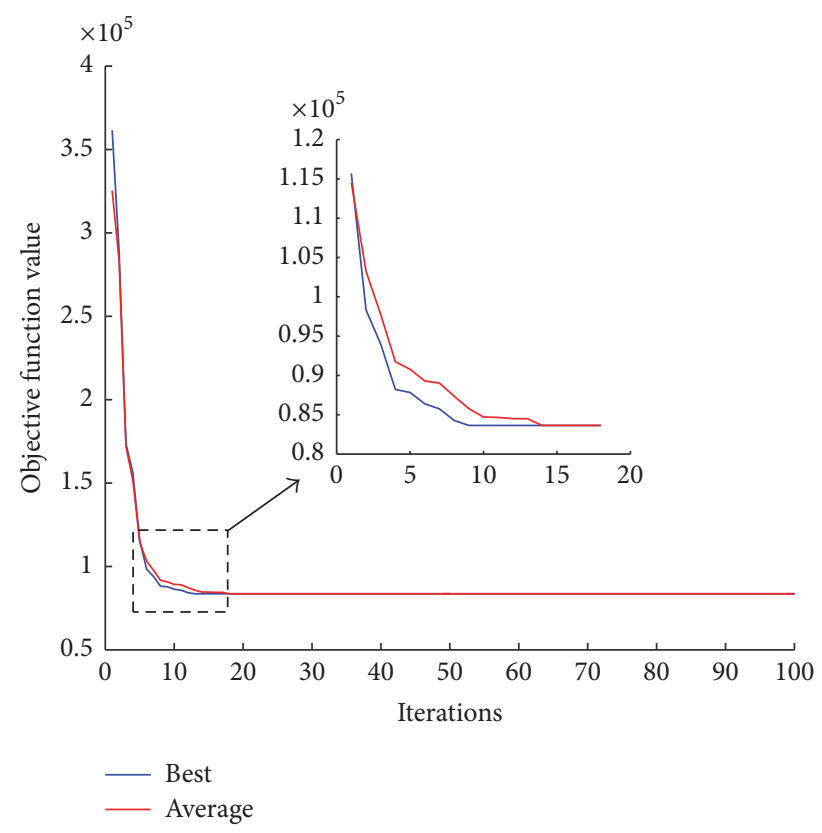

FIGURE 12: The iterative process of IACSA.

example, demands at each level in period 1 are presented in Table 4 in Appendix.

$C_{j s t}$ : it is estimated according to the intercity rail planning presented in Figure 11 and the passenger transport volume of Beijing-Tianjin-Hebei Region from China's Urban Statistical Yearbooks (2015). For example, capacities at each level in period 1 are presented in Table 4 in Appendix.

6.2. Computational Results. The IACSA is coded in C\#, running on a laptop with $2.50 \mathrm{GHz}$ Intel Core I5-2450M processor and $4 \mathrm{~GB}$ of RAM under the system Microsoft Windows 7. Also, the optimization software CPLEX version 12.2 is utilized to solve the problem. The parameters of the IACSA are defined as follows: $M=30, A=10, a_{0}=5$, $\varepsilon_{\text {max }}=0.9, \varepsilon_{\text {min }}=0.1, B=15, b_{0}=5, N=100, Q=10^{6}$, $\delta_{1}=10^{6}, \delta_{2}=10^{6}$, and $\delta_{3}=10^{6}$.

The problem is run 10 times by IACSA. The iterative process of IACSA is presented in Figure 12.

The objective function value and computational time of IACSA are compared with the results from CPLEX, as shown in Table $1 . Z_{\text {CPLEX }}$ is the optimal objective function value of the model, and $Z_{\text {IACSA }}$ is the objective function value of IACSA. Gap is defined as follows:

$$
\text { Gap }=\frac{Z_{\text {IACSA }}-Z_{\text {CPLEX }}}{Z_{\text {CPLEX }}} \times 100 \% .
$$

According to Table 1, Gap between IACSA and CPLEX is less than $1 \%$ for the case. Also, the average computational time of IACSA is shorter than that of CPLEX. For large-size problem, random numbers are generated (see Table 1). In large-size problem, the computational time of CPLEX is more than $10 \mathrm{~h}$. However, IACSA can get the near optimum in half
TABLE 4: Different-level demands and maximum capacities of areas in Beijing-Tianjin-Hebei Region in 2020 (ten thousand persons annually).

\begin{tabular}{|c|c|c|c|c|c|c|}
\hline Area & $q_{i 11}$ & $q_{i 21}$ & $q_{i 31}$ & $C_{j 11}$ & $C_{j 21}$ & $C_{j 31}$ \\
\hline 1 & 40301 & 2529 & 1651 & 61131 & 5227 & 5119 \\
\hline 2 & 57598 & 3615 & 2360 & 87369 & 6142 & 5119 \\
\hline 3 & 172395 & 9306 & 7065 & 261502 & 9648 & 8462 \\
\hline 4 & 101128 & 6347 & 4144 & 153398 & 6534 & 13285 \\
\hline 5 & 28755 & 1805 & 1178 & 43617 & 0 & 0 \\
\hline 6 & 159168 & 7631 & 6523 & 241438 & 8574 & 0 \\
\hline 7 & 32837 & 2061 & 1346 & 49809 & 6403 & 0 \\
\hline 8 & 40057 & 2514 & 1642 & 60762 & 6860 & 5119 \\
\hline 9 & 25249 & 1585 & 1035 & 38299 & 6599 & 9645 \\
\hline 10 & 71147 & 4465 & 2916 & 107920 & 6272 & 0 \\
\hline 11 & 50313 & 3158 & 2062 & 76318 & 6991 & 6399 \\
\hline 12 & 12141 & 810 & 498 & 18417 & 0 & 0 \\
\hline 13 & 11543 & 725 & 473 & 17510 & 0 & 0 \\
\hline 14 & 10670 & 670 & 437 & 16185 & 3920 & 0 \\
\hline 15 & 12187 & 765 & 499 & 18487 & 4247 & 0 \\
\hline 16 & 7312 & 459 & 300 & 11092 & 0 & 0 \\
\hline 17 & 90939 & 5430 & 3415 & 101352 & 5440 & 5701 \\
\hline 18 & 12920 & 686 & 485 & 14399 & 2176 & 2851 \\
\hline 19 & 14584 & 871 & 548 & 16254 & 3264 & 0 \\
\hline 20 & 12774 & 763 & 480 & 14236 & 0 & 0 \\
\hline 21 & 14568 & 870 & 547 & 16236 & 2984 & 0 \\
\hline 22 & 20439 & 1085 & 767 & 22779 & 3228 & 0 \\
\hline 23 & 16224 & 861 & 609 & 18082 & 3264 & 0 \\
\hline 24 & 52152 & 2768 & 1958 & 58124 & 3445 & 5131 \\
\hline 25 & 8552 & 454 & 321 & 9531 & 0 & 0 \\
\hline 26 & 13815 & 733 & 519 & 15397 & 0 & 0 \\
\hline 27 & 16345 & 868 & 614 & 18216 & 0 & 0 \\
\hline 28 & 20616 & 3259 & 1481 & 23344 & 8299 & 0 \\
\hline 29 & 53405 & 8443 & 3837 & 60471 & 19151 & 14505 \\
\hline 30 & 40085 & 6337 & 2880 & 45388 & 12129 & 2872 \\
\hline 31 & 13925 & 2201 & 1000 & 15767 & 2160 & 0 \\
\hline 32 & 37114 & 5868 & 2666 & 42024 & 13086 & 8703 \\
\hline 33 & 44258 & 6997 & 3180 & 50113 & 11810 & 2843 \\
\hline 34 & 29118 & 4603 & 2092 & 32970 & 0 & 0 \\
\hline 35 & 20911 & 3306 & 1502 & 23678 & 3541 & 0 \\
\hline 36 & 14930 & 2360 & 1073 & 16905 & 3126 & 0 \\
\hline 37 & 31108 & 4918 & 2235 & 35224 & 5832 & 0 \\
\hline 38 & 17556 & 2775 & 1261 & 19878 & 2869 & 0 \\
\hline
\end{tabular}

an hour. Gap between IACSA and CPLEX is still less than $1 \%$. Therefore, it can be concluded that IACSA is producing reasonable results in large-size problems.

Figure 13 presents the location of THUAA in BeijingTianjin-Hebei Region. In the short-term period, the location planning of THUAA is consistent with the spatial development pattern, the transportation network, and the population distribution. In the medium-term and long-term periods, along with the development of intercity rail, the increase of urban population and the urban population migration from 


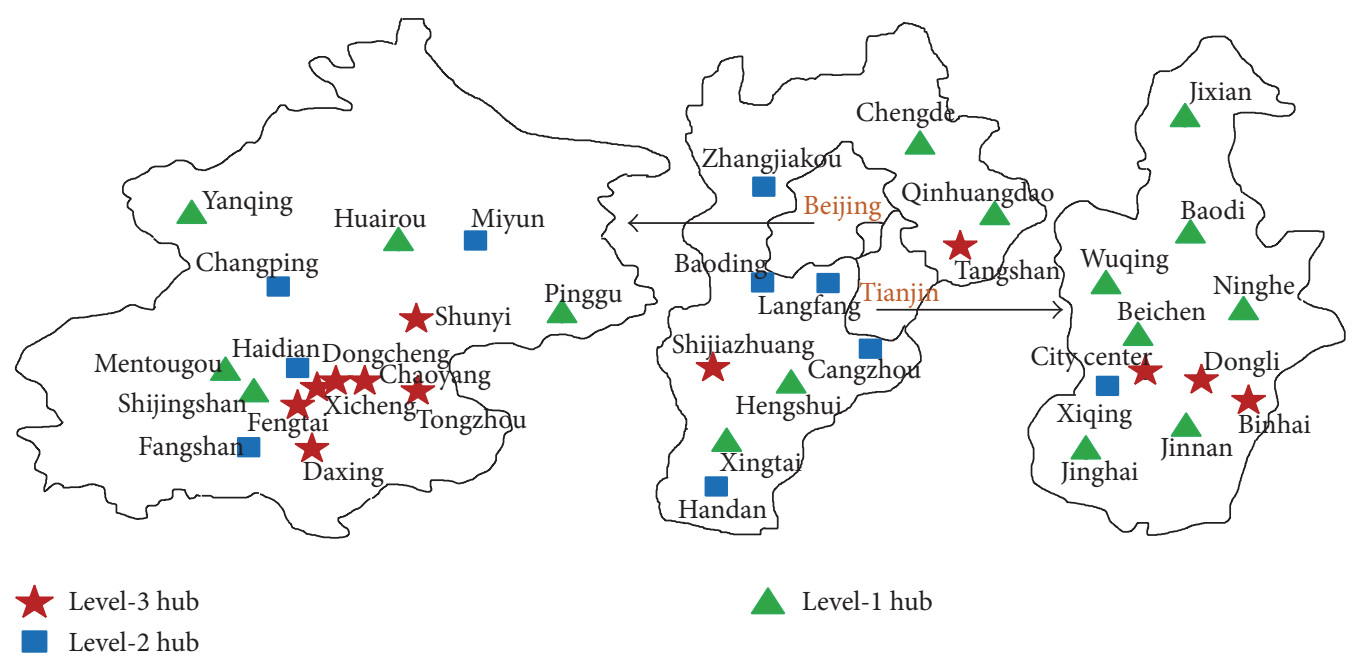

(a) The short-term location planning of THUAA in Beijing-Tianjin-Hebei Region

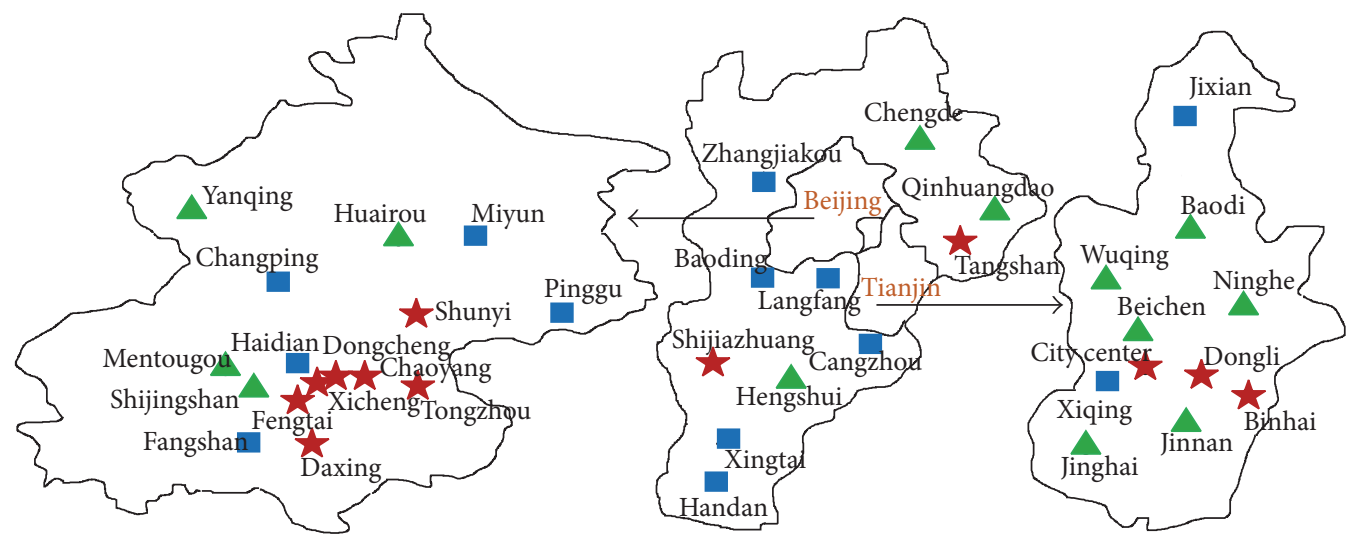

Level-3 hub

$\triangle$ Level-1 hub

Level-2 hub

(b) The medium-term location planning of THUAA in Beijing-Tianjin-Hebei Region

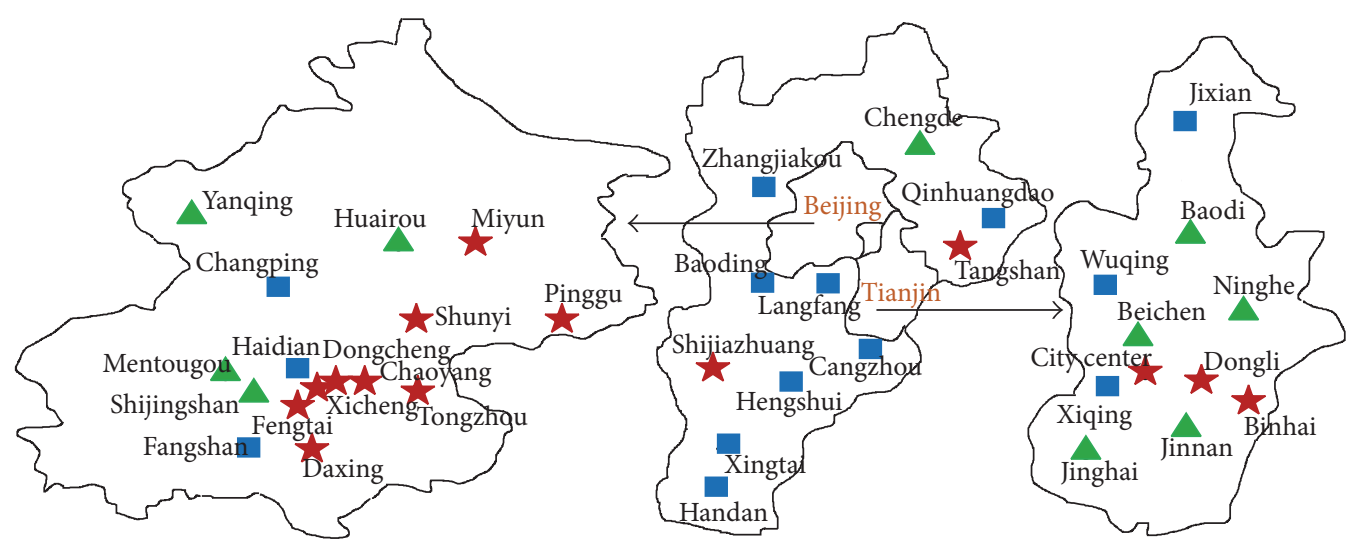

Level-3 hub

Level-1 hub

Level-2 hub

(c) The long-term location planning of THUAA in Beijing-Tianjin-Hebei Region

FIgURE 13: The location of THUAA in Beijing-Tianjin-Hebei Region. 


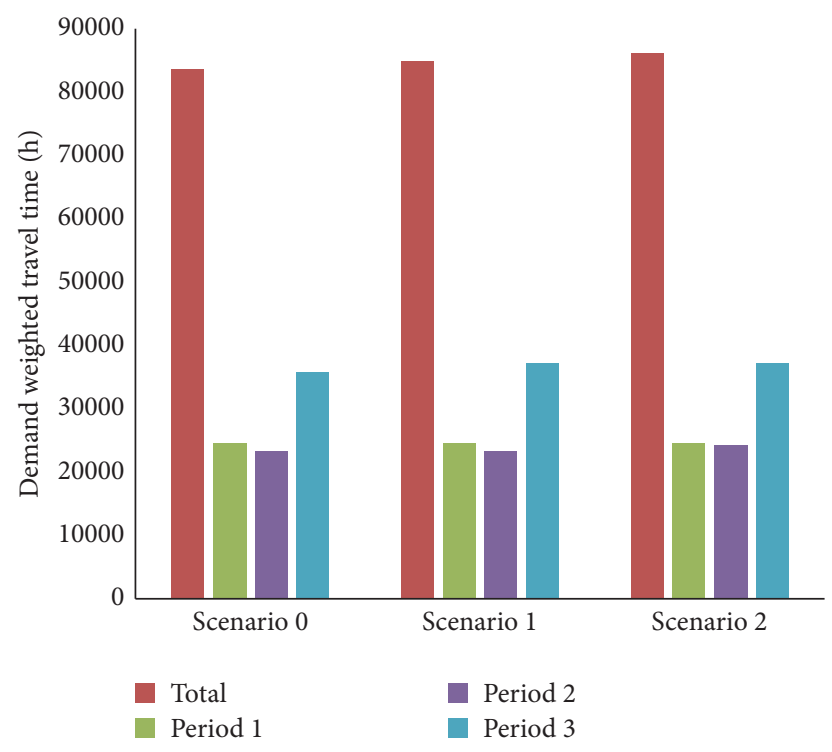

FIGURE 14: The demand weighted travel time in different scenarios.

city center to suburban, more and more level-2 and level-3 hubs are opened.

In addition, 3 scenarios are designed to investigate the impact of urban population migration on the location of THUAA. Scenario 0, the basic scenario with the parameters defined in the previous sections, includes 3 periods of urban population migration. In scenario 1 , urban population migration happens in periods 1 and 2 . In scenario 2, urban population migration happens only in period 1 . Figure 14 shows that the more the urban population migration (from scenario 2 to scenario 0 ) is, the less the total demand weighted travel time is. In Figure 15, we can find that, in all scenarios as time goes, the number of level-1 hub reduces, while the number of level-2 hub increases and the number of level3 hub remains the same or increases. Moreover, in each period, with more and more urban population migration (from scenario 2 to scenario 0), the number of level-1 hub remains the same or becomes lower, while both the numbers of level-2 hub and level-3 hub remain the same or become higher. In all, urban population migration helps to reduce travel time for urban residents, which needs a sustained and appropriate implementation.

\section{Conclusion and Further Research}

In this paper, the changes of passenger demand and the development of transportation network in the process of rapid urbanization are considered and MHLP of THUAA is studied. According to the hierarchical feature of PDUAA and the hierarchical nature of THUAA, a hierarchical service network of THUAA with a multiflow, nested and noncoherent structure is described. To better meet the time-varying demand in the process of rapid urbanization, the multiperiod nature of the hierarchical location problem of THUAA is taken into account. Thus, a mathematical programming model and a solution algorithm for multiperiod location

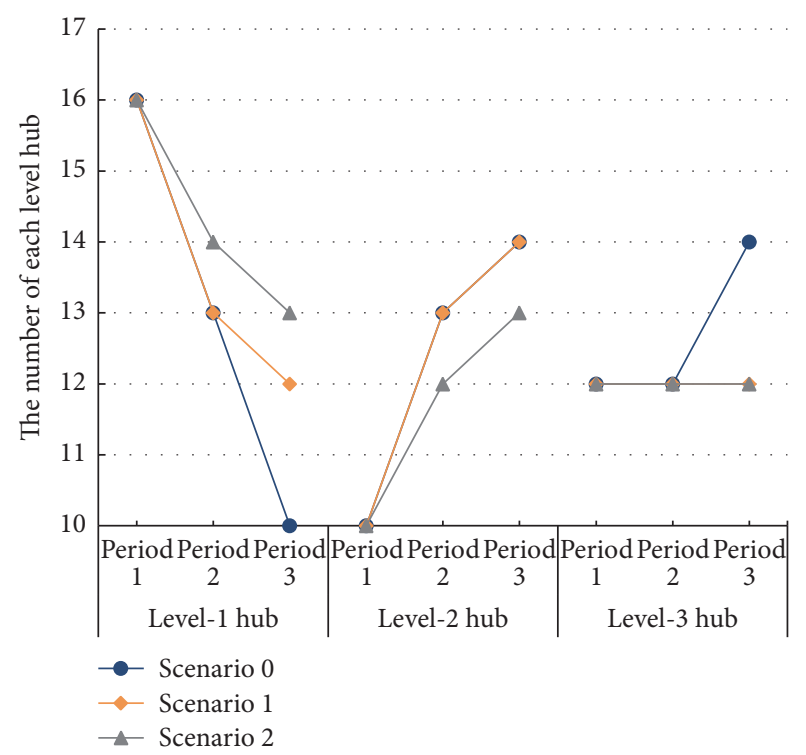

FIGURE 15: The number of hubs at each level in different scenarios.

of THUAA hierarchical system are proposed. The proposed model and algorithm are verified by the application to a reallife problem of Beijing-Tianjin-Hebei Region in China. The results of different scenarios in the case show that urban population migration has a great impact on the hub location scheme. Also, sustained and appropriate urban population migration helps to reduce travel time for urban residents.

Due to the uncertainty of the parameters (e.g., demands at each level, the coverages of different-level hubs, and the maximum capacity of each candidate hub), MHLP of THUAA under uncertainty needs further research in the future.

\section{Appendix}

In Tables 2, 3, and 4, the administrative districts in Beijing (i.e., Dongcheng, Xicheng, Chaoyang, Fengtai, Shijingshan, Haidian, Fangshan, Tongzhou, Shunyi, Changping, Daxing, Mentougou, Huairou, Pinggu, Miyun, and Yanqing) are numbered as $1 \sim 16$, respectively. The administrative districts in Tianjin (i.e., city center, Dongli, Xiqing, Jinnan, Beichen, Wuqing, Baodi, Binhai, Ninghe, Jinghai, and Jixian) are numbered as 17 27, respectively. The 11 cities in Hebei Province (i.e., Langfang, Shijiazhuang, Baoding, Qinhuangdao, Tangshan, Handan, Xingtai, Zhangjiakou, Chengde, Cangzhou, and Hengshui) are numbered as 28 38, respectively.

\section{Competing Interests}

The authors declare that there is no conflict of interests regarding the publication of this paper.

\section{Acknowledgments}

The authors acknowledge the financial support by National Basic Research Program of China (no. 2012CB725403), 
National Natural Science Foundation of China (no. 61374202 and Key Program no. U1434207), and the Fundamental Research Funds for the Central Universities of China (no. 2014JBM067).

\section{References}

[1] R. Z. Farahani, M. Hekmatfar, B. Fahimnia, and N. Kazemzadeh, "Hierarchical facility location problem: models, classifications, techniques, and applications," Computers \& Industrial Engineering, vol. 68, no. 1, pp. 104-117, 2014.

[2] G. Şahin and H. Süral, "A review of hierarchical facility location models," Computers and Operations Research, vol. 34, no. 8, pp. 2310-2331, 2007.

[3] C. Pehlivan, V. Augusto, and X. Xie, "Dynamic capacity planning and location of hierarchical service networks under service level constraints," IEEE Transactions on Automation Science and Engineering, vol. 11, no. 3, pp. 863-880, 2014.

[4] J. Baray and G. Cliquet, "Optimizing locations through a maximum covering/p-median hierarchical model: maternity hospitals in France," Journal of Business Research, vol. 66, no. 1, pp. 127-132, 2013.

[5] A. M. Mestre, M. D. Oliveira, and A. Barbosa-Povoa, "Organizing hospitals into networks: a hierarchical and multiservice model to define location, supply and referrals in planned hospital systems," OR Spectrum, vol. 34, no. 2, pp. 319-348, 2012.

[6] J. C. Teixeira and A. P. Antunes, "A hierarchical location model for public facility planning," European Journal of Operational Research, vol. 185, no. 1, pp. 92-104, 2008.

[7] M. M. L. Nappi and J. C. Souza, "Disaster management: hierarchical structuring criteria for selection and location of temporary shelters," Natural Hazards, vol. 75, no. 3, pp. 2421-2436, 2015.

[8] Z. F. Chen, X. Chen, Q. Li, and J. Chen, “The temporal hierarchy of shelters: a hierarchical location model for earthquake-shelter planning," International Journal of Geographical Information Science, vol. 27, no. 8, pp. 1612-1630, 2013.

[9] A. I. Barros, R. Dekker, and V. Scholten, "A two-level network for recycling sand: a case study," European Journal of Operational Research, vol. 110, no. 2, pp. 199-214, 1998.

[10] Y. Yan, B. Zhang, J. Zheng, and J. Ma, "Hierarchical location service for wireless sensor networks with mobile sinks," Wireless Communications \& Mobile Computing, vol. 10, no. 7, pp. 899-911, 2010.

[11] A. A. V. Ignacio, V. J. M. F. Filho, and R. D. Galvão, "Lower and upper bounds for a two-level hierarchical location problem in computer networks," Computers \& Operations Research, vol. 35, no. 6, pp. 1982-1998, 2008.

[12] D. Serra and C. ReVelle, "The pq-median problem: location and districting of hierarchical facilities. Part ii: heuristic solution methods," Location Science, vol. 2, no. 2, 1994.

[13] M. A. Drexl, "An approximation algorithm for the $k$-level concentrator location problem," Operations Research Letters, vol. 39, no. 5, pp. 355-358, 2011.

[14] A. F. Gabor and J.-K. C. van Ommeren, "A new approximation algorithm for the multilevel facility location problem," Discrete Applied Mathematics, vol. 158, no. 5, pp. 453-460, 2010.

[15] J. M. Lee and Y. H. Lee, "Tabu based heuristics for the generalized hierarchical covering location problem," Computers \& Industrial Engineering, vol. 58, no. 4, pp. 638-645, 2010.
[16] A. B. Arabani and R. Z. Farahani, "Facility location dynamics: an overview of classifications and applications," Computers \& Industrial Engineering, vol. 62, no. 1, pp. 408-420, 2012.

[17] T. S. Hale and C. R. Moberg, "Location science research: a review," Annals of Operations Research, vol. 123, no. 1-4, pp. 2135, 2003.

[18] T. Wu, H. Shen, and C. Zhu, "A multi-period location model with transportation economies-of-scale and perishable inventory," International Journal of Production Economics, vol. 169, pp. 343-349, 2015.

[19] B. Zahiri, R. Tavakkoli-Moghaddam, and M. S. Pishvaee, "A robust possibilistic programming approach to multi-period location-allocation of organ transplant centers under uncertainty," Computers \& Industrial Engineering, vol. 74, no. 1, pp. 139-148, 2014.

[20] M. Albareda-Sambola, A. Alonso-Ayuso, L. F. Escudero, E. Fernández, and C. Pizarro, "Fix-and-relax-coordination for a multi-period location-allocation problem under uncertainty," Computers \& Operations Research, vol. 40, no. 12, pp. 28782892, 2013.

[21] M. Albareda-Sambola, A. Alonso-Ayuso, L. F. Escudero et al., "A computational comparison of several formulations for the multi-period incremental service facility location problem," Top, vol. 18, no. 1, pp. 62-80, 2010.

[22] Y. Hinojosa, J. Puerto, and F. R. Fernández, "A multiperiod twoechelon multicommodity capacitated plant location problem," European Journal of Operational Research, vol. 123, no. 2, pp. 271-291, 2000.

[23] X.-F. Wang, X.-M. Sun, and Y. Fang, "Genetic algorithm solution for multi-period two-echelon integrated competitive/uncompetitive facility location problem," Asia-Pacific Journal of Operational Research, vol. 25, no. 1, pp. 33-56, 2008.

[24] A. Antunes, O. Berman, J. Bigotte, and D. Krass, "A location model for urban hierarchy planning with population dynamics," Environment and Planning A, vol. 41, no. 4, pp. 996-1016, 2009.

[25] S. M. Garrett, "Parameter-free, adaptive clonal selection," in Proceedings of the Congress on Evolutionary Computation (CEC '04), pp. 1052-1058, Portland, Ore, USA, June 2004.

[26] B. H. Ulutas and S. Kulturel-Konak, "A review of clonal selection algorithm and its applications," Artificial Intelligence Review, vol. 36, no. 2, pp. 117-138, 2011.

[27] D. Bertsimas and J. Tsitsiklis, "Simulated annealing," Statistical Science, vol. 8, no. 1, pp. 10-15, 1993. 


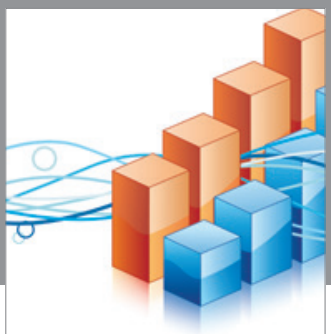

Advances in

Operations Research

vatem alat4

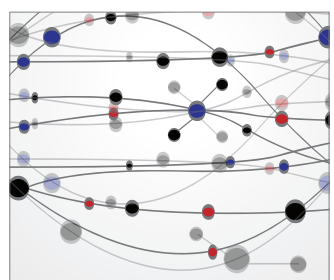

\section{The Scientific} World Journal
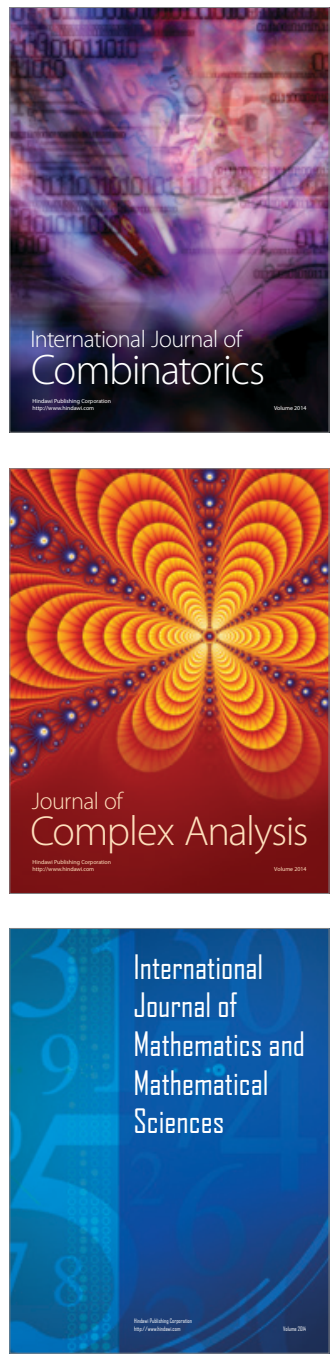
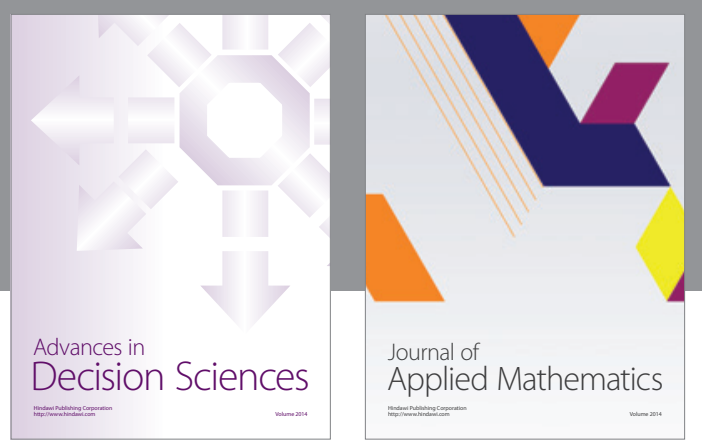

Algebra

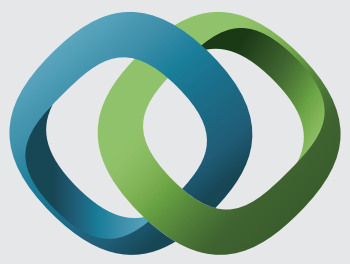

\section{Hindawi}

Submit your manuscripts at

https://www.hindawi.com
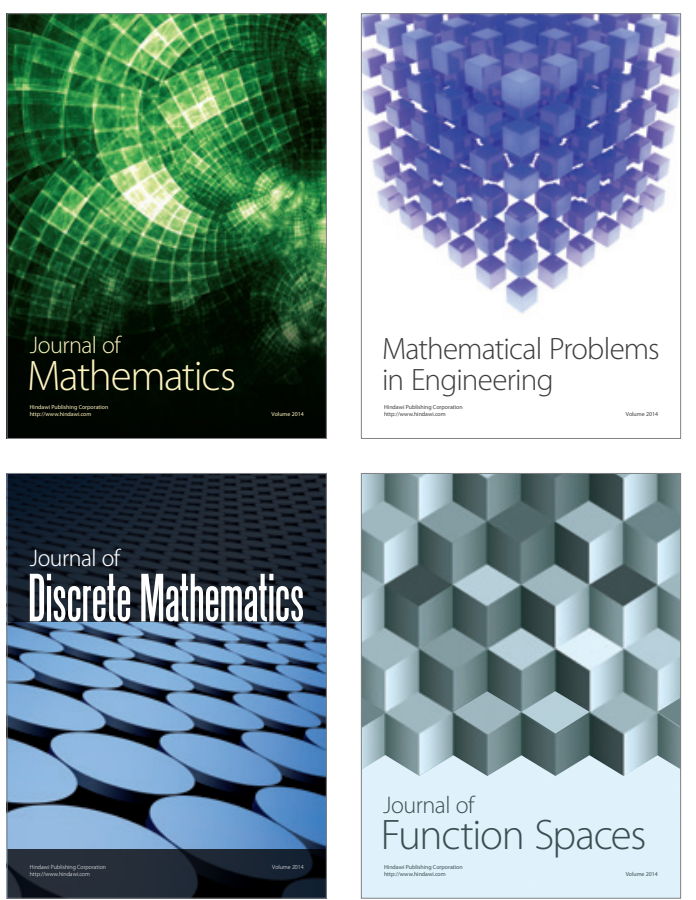

Mathematical Problems in Engineering
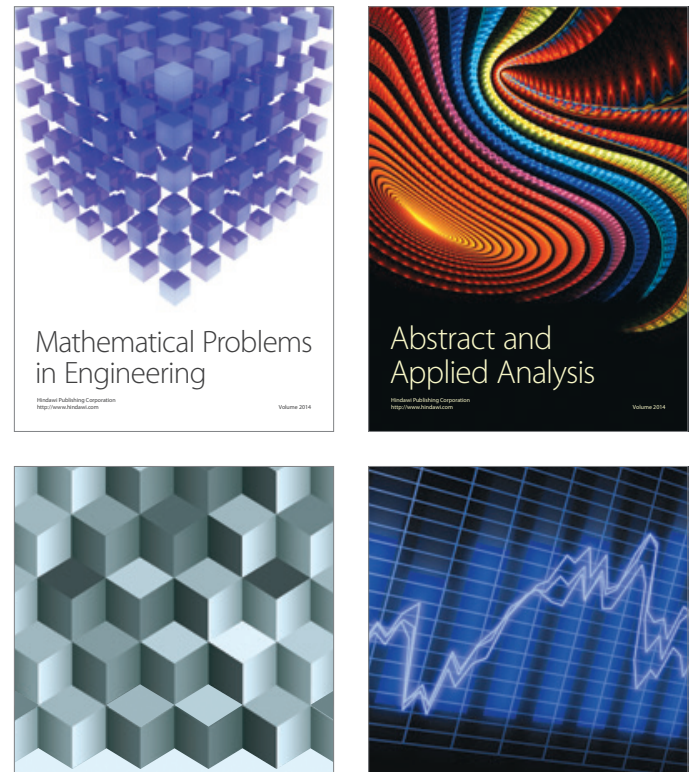

Journal of

Function Spaces

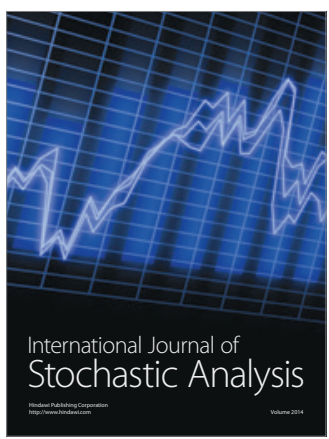

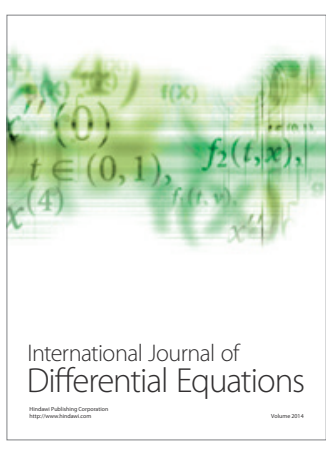
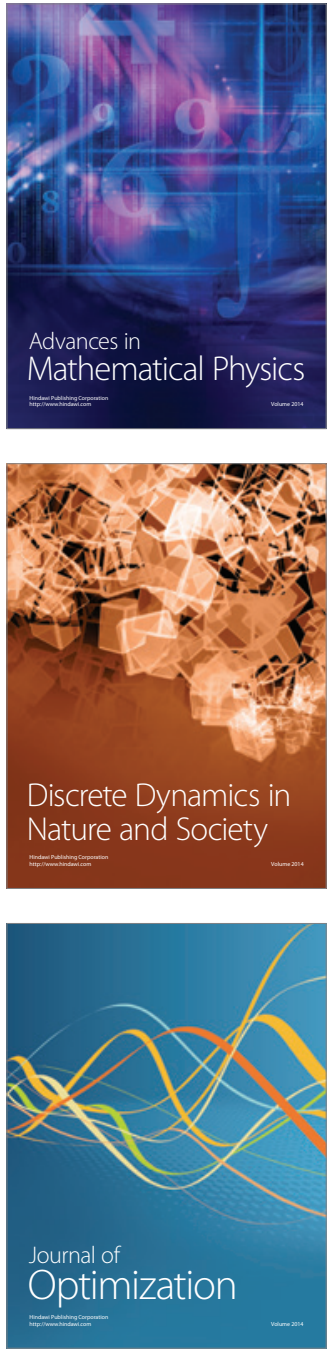